IFIC-07-74, FTUV-07-1207

FERMILAB-PUB-07-643-T

\title{
Light charged Higgs at the beginning of the LHC era
}

\author{
Gabriela Barenboim * Paride Paradisi $\dagger$ and Oscar Vive $\sharp$ \\ Departament de Física Teòrica and IFIC, \\ Universitat de València-CSIC, E-46100, Burjassot, Spain. \\ Enrico Lunghi $\$$ \\ Fermi National Accelerator Laboratory, \\ P.O. Box 500, Batavia, IL 60510-0500, USA. \\ Werner Porod \\ Institut für Theoretische Physik und Astrophysik, \\ Universität Würzburg, D-97074 Würzburg, Germany.
}

\begin{abstract}
The terascale will be explored with the start of the LHC. One of the most fundamental questions which we expect to be answered is the root of electroweak symmetry breaking and whether the Higgs mechanism is realized in nature or not. In this context we pose the question if existing experimental data still allow for a light non-minimal Higgs sector. We tackle this question first in the context of the two Higgs doublet model and then we concentrate in two supersymmetric models, the constrained MSSM and the MSSM with non-universal Higgs masses. In both supersymmetric scenarios, light pseudoscalar and light charged-Higgs bosons are still viable provided $\tan \beta$ is large. In this regime, we emphasize the importance of the constraints provided by the decay $B \rightarrow \tau \nu$ mediated by the charged-Higgs at tree-level. In addition we comment on generic predictions for hadronic colliders and indirect searches in such scenarios.
\end{abstract}

*Electronic address: gabriela.barenboim@uv.es

$\dagger$ Electronic address: paride.paradisi@uv.es

${ }^{\ddagger}$ Electronic address: oscar.vives@uv.es

$\S$ Electronic address: lunghi@fnal.gov

`Electronic address: porod@physik.uni-wuerzburg.de 


\section{INTRODUCTION}

The presence of a non-standard Higgs boson with a "small" mass, below $200 \mathrm{GeV}$, would be a very interesting possibility in the first years of LHC operation. In fact, the interest on this possibility has been recently increased with the small differences from Standard Model (SM) expectations found at CDF and D0 [1, 2] and has motivated several analysis in the context of the minimal supersymmetric standard model (MSSM) [3, 4]. Even though the results are completely compatible with the absence of non-SM Higgs bosons at the $2 \sigma$ level these small discrepancies have motivated the question whether it is possible to have a light non-SM Higgs consistent with the present experimental constraints. In this letter we intend to answer this question in models with 2 Higgs doublets and specially in the framework of the MSSM. During the first years of LHC operation and with the new measurements at Tevatron, top quark physics will receive a big boost with a significantly improved understanding of its physics and perhaps find a first clue of physics beyond the SM. Perhaps the best possible situation to obtain sizeable beyond-the-SM effects in top-quark physics corresponds to the existence of a charged Higgs boson of mass close to the top quark mass. In this work we will explore the possibility of having such a light Higgs sector in different models and how this affects phenomenology.

Clearly, the presence of a charged Higgs implies necessarily an extended Higgs sector. Therefore the simplest model we can explore and our first option is a two Higgs doublet model (2HDM). In a generic type II $2 \mathrm{HDM}$ we see that the charged-Higgs is constrained to be heavier than $295 \mathrm{GeV}$ by $\mathrm{BR}(b \rightarrow s \gamma)$, although a pseudoscalar mass in the range 150-200 GeV is still allowed. As a second option we consider supersymmetric models, where we find that a light charged-Higgs below $200 \mathrm{GeV}$ is still possible both in the Constrained MSSM (CMSSM) and in an MSSM with non-universal Higgs masses. However, in these models the decay $B \rightarrow \tau \nu$ is a very strong constraint in the light $m_{H^{+}}$-large $\tan \beta$ region and, in particular, in the CMSSM sets a strict lower limit of $180 \mathrm{GeV}$ for the charged-Higgs mass.

In the next section we explore in detail a generic type II two Higgs doublet model. Section III analyzes the CMSSM and a MSSM with non-universal Higgs masses and comments about models with mediation mechanisms other than gravity. In section [V] we present the signatures of the light charged-Higgs scenario in collider and indirect search experiments. 
Finally in section $\square$ we present our conclusions.

\section{TWO HIGGS DOUBLET MODELS}

The two Higgs doublet model is the simplest extension of the SM obtained with the only addition of a second Higgs doublet. A 2HDM with generic Yukawa couplings has severe Flavour Changing Neutral Currents (FCNC) problems and, for this reason, the Higgs couplings are restricted by an ad hoc discrete symmetry to forbid FCNC at tree-level. The two main options are the type-I and the type-II 2HDMs, depending on whether the up-type and down-type fermions are coupled to the same or different Higgs doublets respectively. In our analysis, we will assume a type-II 2HDM with a Higgs potential given by [5]

$$
\begin{aligned}
V_{\mathrm{THDM}}= & m_{1}^{2}\left|\Phi_{1}\right|^{2}+m_{2}^{2}\left|\Phi_{2}\right|^{2}-m_{3}^{2}\left(\Phi_{1}^{\dagger} \Phi_{2}+\Phi_{2}^{\dagger} \Phi_{1}\right)+\frac{\lambda_{1}}{2}\left|\Phi_{1}\right|^{4}+\frac{\lambda_{2}}{2}\left|\Phi_{2}\right|^{4} \\
& +\lambda_{3}\left|\Phi_{1}\right|^{2}\left|\Phi_{2}\right|^{2}+\lambda_{4}\left|\Phi_{1}^{\dagger} \Phi_{2}\right|^{2}+\frac{\lambda_{5}}{2}\left\{\left(\Phi_{1}^{\dagger} \Phi_{2}\right)^{2}+\left(\Phi_{2}^{\dagger} \Phi_{1}\right)^{2}\right\}
\end{aligned}
$$

where $\Phi_{i}$ are the Higgs iso-doublets with hypercharge $\frac{1}{2}$. Being a type-II 2HDM, this potential satisfies a (softly-broken) discrete symmetry under the transformation $\Phi_{1} \rightarrow \Phi_{1}$ and

$\Phi_{2} \rightarrow-\Phi_{2}$. A nonzero value of $m_{3}^{2}$ indicates that the discrete symmetry is broken softly and would correspond to a $B \mu$ coupling in supersymmetric models. The eight free parameters $\left(m_{1}^{2}-m_{3}^{2}\right.$ and $\left.\lambda_{1}-\lambda_{5}\right)$ can be rewritten in terms of eight "physical" parameters, i.e. four Higgs mass parameters $m_{h}, m_{H}, m_{A}, m_{H^{ \pm}}$, two mixing angles $\alpha, \beta$, the vacuum expectation value $v$, and the soft-breaking scale of the discrete symmetry $M$. The two physical CP-even fields $h$ and $H$ are such that $m_{h}^{0} \leq m_{H}^{0}$. In particular, the masses of the CP-odd, $A$ (CP-odd) and the charged Higgs, $H^{ \pm}$, are related by the following expression

$$
m_{H^{ \pm}}^{2}-m_{A}^{2}=\frac{1}{2}\left(\lambda_{5}-\lambda_{4}\right) v^{2}
$$

with $v=\sqrt{v_{1}^{2}+v_{2}^{2}}$, where $v_{1}$ and $v_{2}$ are the vacuum expectation values of $\Phi_{1}$ and $\Phi_{2}$, respectively. Imposing the vacuum conditions we can replace $m_{1}^{2}$ and $m_{2}^{2}$ by $v_{1}$ and $v_{2}$. Then the masses of the heavier bosons $\left(H, H^{ \pm}\right.$and $\left.A\right)$ take the form $m_{\Phi}^{2}=m_{3}^{2} /(\sin \beta \cos \beta)^{2}+\lambda_{i} v^{2}$, where $\lambda_{i}$ is a linear combination of $\lambda_{1}-\lambda_{5}$. When $m_{3}^{2} /(\sin \beta \cos \beta)^{2} \gg \lambda_{i} v^{2}$, the mass $m_{\Phi}^{2}$ is determined by the soft-breaking scale of the discrete symmetry $m_{3}^{2}$, and is independent of $\lambda_{i}$. This corresponds to the so-called decoupling limit. On the contrary, when $M^{2}$ is limited to be at the weak scale $\left(M^{2} \lesssim \lambda_{i} v^{2}\right)$ a large value of $m_{\Phi}$ is realized by taking $\lambda_{i}$ to be large; i.e., 
the strong coupling regime. However, too large $\lambda_{i}$ leads to the breakdown of perturbation theory [6, 7, 8]. Furthermore, low energy precision data also impose important constraints on the model parameters [9]. We take into account the following bounds to constrain the 2 HDM parameters ${ }^{1}$ :

i) Perturbative unitarity [6], corresponding to $\left|a^{0}\left(\varphi_{A} \varphi_{B} \rightarrow \varphi_{C} \varphi_{D}\right)\right|<\xi$ (we take $\xi=1 / 2$ in our analysis), where $a^{0}\left(\varphi_{A} \varphi_{B} \rightarrow \varphi_{C} \varphi_{D}\right)$ is the S-wave amplitude for the elastic scattering process $\varphi_{A} \varphi_{B} \rightarrow \varphi_{C} \varphi_{D}$ of the longitudinally polarized gauge bosons (and Higgs bosons). These conditions translate into constraints on the couplings $\lambda_{i}(i=1-5)$ [7, 8].

ii) Vacuum stability [11]

iii) Constraints on oblique-corrections from LEP with the $S, T$ and $U$ parameters [9]. In particular, the $T$ parameter is such that $T \simeq \alpha_{E M}^{-1} \Delta \rho$, with $\Delta \rho \leq 10^{-3}$. The above constraint can be satisfied if a custodial $S U(2)_{V}[12]$ is approximately conserved and this happens if (1) $m_{H^{ \pm}} \simeq m_{A}$, and $(2) m_{H^{ \pm}} \simeq m_{H}$ with $\sin ^{2}(\alpha-\beta) \simeq 1$ or $m_{H^{ \pm}} \simeq m_{h}$ with $\cos ^{2}(\alpha-\beta) \simeq 1[12,13]$

iv) B-physics constraints, in particular $\bar{B} \rightarrow X_{s} \gamma$ and $B \rightarrow \tau \nu$. Regarding $\bar{B} \rightarrow X_{s} \gamma$, the present experimental world average performed by HFAG [14] is

$$
\mathcal{B}\left(\bar{B} \rightarrow X_{s} \gamma\right)_{\exp }=\left(3.55 \pm 0.24_{-0.10}^{+0.09} \pm 0.03\right) \times 10^{-4}
$$

while the theoretical estimate performed at the NNLO level [15, 16] (for the reference value $\left.E_{\text {cut }}=1.6 \mathrm{GeV}\right)$ is

$$
\mathcal{B}\left(\bar{B} \rightarrow X_{s} \gamma\right)_{\mathrm{SM}}=(3.15 \pm 0.23) \times 10^{-4}
$$

The NNLO SM prediction for $\mathcal{B}\left(\bar{B} \rightarrow X_{s} \gamma\right)_{\mathrm{SM}}$ is lower than $\mathcal{B}\left(\bar{B} \rightarrow X_{s} \gamma\right)_{\text {exp }}$ by more than $1 \sigma$. This fact allows sizable NP contributions with the same sign as the SM ones like charged-Higgs boson contributions in 2HDMs. In the numerics we utilize the

\footnotetext{
${ }^{1}$ For comparison, see the recent analysis of Ref. [10]. Even if our results qualitatively agree with those of Ref. 10], our numerical analysis was still necessary to understand whether the specific scenario studied in the present work is possible within a $2 \mathrm{HDM}$.
} 
formulae presented in Ref. [17] and updated in Ref. [18] that take into account the NNLO contributions for the SM [15, 16].

Combining the SM prediction and the experimental results for $\mathcal{B}\left(\bar{B} \rightarrow X_{s} \gamma\right)$, we impose the constraint

$$
R_{b s \gamma}^{\exp }=\frac{\mathcal{B}^{\exp }(b \rightarrow s \gamma)}{\mathcal{B}^{\mathrm{SM}}(b \rightarrow s \gamma)}=1.13 \pm 0.12
$$

at the $2 \sigma$ level.

Combining the recent $B$-factory results [19, 20], with the SM expectation $\mathcal{B}\left(B_{u} \rightarrow \tau \nu\right)^{\mathrm{SM}}=G_{F}^{2} m_{B} m_{\tau}^{2} f_{B}^{2}\left|V_{u b}\right|^{2}\left(1-m_{\tau}^{2} / m_{B}^{2}\right)^{2} /\left(8 \pi \Gamma_{B}\right)$, whose numerical value suffers from sizable parametrical uncertainties induced by $f_{B}$ and $V_{u b}$, it is found that

$$
R_{B \tau \nu}^{\exp }=\frac{\mathcal{B}^{\exp }\left(B_{u} \rightarrow \tau \nu\right)}{\mathcal{B}^{\operatorname{SM}}\left(B_{u} \rightarrow \tau \nu\right)}=1.07 \pm 0.42
$$

where we have assumed $f_{B}=0.216 \pm 0.022$ and $V_{u b}=(4.00 \pm 0.26) \times 10^{-3}$ (from the average of inclusive and exclusive semileptonic B decay modes) by HFAG [14]. The decay $B_{u} \rightarrow \tau \nu$ represents a very powerful probe of the scenario of light charged Higgs $[21,22,23]$ because it is a tree-level process and we have that

$$
R_{B \tau \nu}=\frac{\mathcal{B}^{2 \mathrm{HDM}}\left(B_{u} \rightarrow \tau \nu\right)}{\mathcal{B}^{\mathrm{SM}}\left(B_{u} \rightarrow \tau \nu\right)}=\left[1-\left(\frac{m_{B}^{2}}{m_{H^{ \pm}}^{2}}\right) \tan ^{2} \beta\right]^{2}
$$

In the case of the decay $B_{s} \rightarrow \mu^{+} \mu^{-}$in a $2 \mathrm{HDM}$ we have $B R\left(B_{s} \rightarrow \mu^{+} \mu^{-}\right)_{2 H D M} \propto$ $\tan ^{4} \beta / M_{H^{ \pm}}^{4}$ [25] instead of $B R\left(B_{s} \rightarrow \mu^{+} \mu^{-}\right)_{S U S Y} \propto \tan ^{6} \beta / M_{A^{0}}^{4}$ that is obtained in SUSY models (see next section). Therefore $B_{s} \rightarrow \mu^{+} \mu^{-}$does not provide a further constraint on the $2 \mathrm{HDM}$ parameter space once the previous constraints are satisfied.

Applying these constraints we have numerically found the allowed range for $m_{H^{+}}$requiring a pseudoscalar mass $m_{A}$ in a narrow region, $150<m_{A} / \mathrm{GeV}<200$, while all the other parameters of the model including $\tan \beta$ are left free. The upper bound on the $m_{H^{+}}$mass (for the imposed range of $m_{A}$ ) is found to be $\sim 400 \mathrm{GeV}$ for any $\tan \beta$ value by the unitarity and $\Delta \rho$ constraints. The lower bound on $M_{H^{ \pm}}$is set by the constraints arising from $\bar{B} \rightarrow X_{s} \gamma$ $\left(M_{H^{ \pm}}>295 \mathrm{GeV}\right.$ at $95 \%$ confidence level independently of $\left.\tan \beta[16]\right)$. This bound is improved for large $\tan \beta$ values $(\tan \beta \sim 45-65)$ by the $B \rightarrow \tau \nu$ constraints. In fact, as discussed in the next section, the tree-level decay $B \rightarrow \tau \nu$ sets a bound on $\tan \beta / M_{H^{+}}$that roughly allows charged-Higgs masses higher than $295 \mathrm{GeV}$ for $\tan \beta=45$ and higher than 
$420 \mathrm{GeV}$ for $\tan \beta=65^{2}$. Therefore a generic $2 \mathrm{HDM}$ of type II can still be compatible with a range between 150 and $200 \mathrm{GeV}$ for the mass of the light pseudoscalar Higgs boson, although the charged Higgs is always constrained to be above $295 \mathrm{GeV}$ by $\bar{B} \rightarrow X_{s} \gamma$.

However, we have to recall that it is very difficult to accommodate the present discrepancy for the muon anomalous magnetic moment in a $2 \mathrm{HDM}$ scenario.

Notice that a scenario with a light pseudoscalar Higgs boson with mass $M_{A} \leq 200 \mathrm{GeV}$ and a charged Higgs with mass $M_{H^{ \pm}} \geq 300 \mathrm{GeV}$ is not compatible with minimal SUSY frameworks.

\section{SUPERSYMMETRIC MODELS}

The Higgs sector of the MSSM is a special case of Eq. (1). The MSSM has been extensively studied in the literature (see e.g. [26, 27] and references therein) and the presence of the different supersymmetric partners of the SM particles increases the phenomenological constraints to satisfy [28]. Therefore, the first question we have to answer is whether it is possible or not to obtain a pseudoscalar Higgs boson of a mass below $200 \mathrm{GeV}$ in the MSSM satisfying simultaneously all the different constraints. We will answer this question basically in two versions of the MSSM: the Constrained MSSM (CMSSM) and the nonuniversal Higgs mass (NUHM) MSSM [29, 30, 31, 32, 33, 34, 35, 36]. Then we comment about other SUSY-breaking mediation mechanisms such as gauge mediation and anomaly mediation models.

\section{A. CMSSM and NUHM}

The CMSSM is fixed by 4 initial parameters : $m_{0}, M_{1 / 2}, A_{0}$ and $\tan \beta$ plus the sign of the $\mu$ parameter. However, the sign of the $\mu$ parameter is bound to be positive by the requirement of a correct prediction to the muon anomalous magnetic moment and the $B \rightarrow X_{s} \gamma$ branching ratio. Before considering in detail the different indirect constraints it is useful to identify the possible regions of CMSSM parameter space that can accommodate

\footnotetext{
${ }^{2}$ In fact, lighter charged Higgs masses than these values, although never lighter than $295 \mathrm{GeV}$, can be allowed for larger $\tan \beta$ values if the SM contribution is canceled by a charged Higgs contribution as large as twice the SM one with opposite sign.
} 
a pseudoscalar mass below $200 \mathrm{GeV}$.

In order to establish the $m_{H^{+}}^{2}, m_{A}^{2}$ dependence on the input parameters of the CMSSM, let us consider the following tree-level expressions and their approximate values at medium to large values of $\tan \beta$ :

$$
\begin{aligned}
\mu^{2} & =\frac{m_{H_{d}}^{2}\left(m_{t}\right)-m_{H_{u}}^{2}\left(m_{t}\right) \tan ^{2} \beta}{\tan ^{2} \beta-1}-\frac{M_{Z}^{2}}{2} \simeq-m_{H_{u}}^{2}\left(m_{t}\right)-\frac{M_{Z}^{2}}{2} \\
m_{A}^{2} & =m_{H_{d}}^{2}\left(m_{t}\right)+m_{H_{u}}^{2}\left(m_{t}\right)+2 \mu^{2} \simeq m_{H_{d}}^{2}\left(m_{t}\right)-m_{H_{u}}^{2}\left(m_{t}\right)-M_{Z}^{2}
\end{aligned}
$$

The mass of the charged Higgs and the pseudoscalar ${ }^{3}$ are very similar in the MSSM, as at tree-level they satisfy the relation:

$$
m_{H^{+}}^{2}=m_{A}^{2}+m_{W}^{2}
$$

These masses are then obtained from the electroweak values $m_{H_{u}}^{2}\left(m_{t}\right)$ and $m_{H_{d}}^{2}\left(m_{t}\right)$ which are determined through the RGEs

$$
\begin{aligned}
16 \pi^{2} \frac{d}{d t} m_{H_{u}}^{2} & \simeq 6 X_{t}-6 g_{2}^{2} M_{1 / 2}^{2} \\
16 \pi^{2} \frac{d}{d t} m_{H_{d}}^{2} & \simeq 6 X_{b}+2 X_{\tau}-6 g_{2}^{2} M_{1 / 2}^{2}
\end{aligned}
$$

with $X_{f}=y_{f}^{2}\left(m_{H}^{2}+m_{\tilde{f}_{L}}^{2}+m_{\tilde{f}_{R}}^{2}+A_{f}^{2}\right)(f=t, b, \tau)$. As it is well known, $m_{H_{u}}^{2}\left(m_{t}\right)<0$ in the entire SUSY parameter space due to the large RGE effects proportional to $y_{t}^{2}$. The approximate numerical solution for $m_{H_{u}}^{2}\left(m_{t}\right)$, valid for any $\tan \beta$ value, is [30]

$$
m_{H_{u}}^{2}\left(m_{t}\right) \simeq-0.12 m_{0}^{2}-2.7 M_{1 / 2}^{2}+0.4 A_{0} M_{1 / 2}-0.1 A_{0}^{2}
$$

clearly showing that $m_{H_{u}}^{2}\left(m_{t}\right)<0$. On the contrary, $m_{H_{d}}^{2}\left(m_{t}\right)$ crucially depends on $\tan \beta$. For instance, if we assume low $/$ moderate $\tan \beta$, i.e. $\tan \beta \leq 10$, we can neglect to first approximation $X_{b, \tau}$ in Eq. (12) and the LO solution for $m_{H_{d}}^{2}\left(m_{t}\right)$ is $m_{H_{d}}^{2}\left(m_{t}\right) \simeq m_{0}^{2}+$ $0.5 M_{1 / 2}^{2}$. In this regime, $m_{H_{d}}^{2}\left(m_{t}\right)>0$ and thus both $m_{H_{d}}^{2}\left(m_{t}\right)$ and $m_{H_{u}}^{2}\left(m_{t}\right)$ provide positive contributions to $m_{A}^{2}$ in Eq. (9). For larger $\tan \beta$ values, negative RGE effects proportional to $y_{b, \tau}^{2}$ reduce $m_{H_{d}}^{2}\left(m_{t}\right)$ until the limit case where $m_{H_{d}}^{2} \simeq m_{H_{u}}^{2}<0$ when $y_{b}^{2} \sim y_{t}^{2}$. In this large $\tan \beta$ regime, $m_{H_{d}}^{2}$ and $m_{H_{u}}^{2}$ provide opposite contributions to $m_{A}^{2}$ in Eq. (9) that, indeed, can result strongly reduced. As we will see below, our numerical analysis confirms that we find light pseudoscalar masses, $m_{A} \leq 200 \mathrm{GeV}$, only for $\tan \beta>50$.

\footnotetext{
${ }^{3}$ Here we are considering a real MSSM with zero phases in the $\mu$ and trilinear parameters.
} 
At large $\tan \beta$, the requirement of correct EW symmetry breaking and a neutral LSP sets important constraints on the allowed $\left(m_{0}, M_{1 / 2}, \tan \beta\right)$ values. In particular in CMSSM models, the lightest stau mass is $M_{\tilde{\tau}_{1}}^{2} \sim M_{\tilde{\tau}_{R}}^{2}-m_{\tau} \mu \tan \beta$ where at leading $\log , M_{\tilde{\tau}_{R}}^{2} \simeq$ $\left.m_{0}^{2}-0.36\left(3 m_{0}^{2}+A_{0}^{2}\right) y_{\tau}^{2}\right]+0.13 M_{1 / 2}^{2}$ and $y_{\tau}=m_{\tau} \tan \beta \sqrt{2} / v($ with $v=246 \mathrm{GeV})$. Thus, the LSP condition $M_{\tilde{\tau}_{1}}>M_{\chi_{1}^{0}}$ generates a lower bound for $m_{0}$ that increases with increasing $\tan \beta$. For instance, at $\tan \beta=50$ the minimum value of $m_{0}$ is $200 \mathrm{GeV}$ for $m_{1 / 2}=200$ $\mathrm{GeV}$. However, the $m_{0}^{2}$ dependence of $m_{A}^{2}$ is quite mild, as a result of the large negative RGE effects driven by $y_{t}^{2}, y_{b}^{2} \sim 1$. So, at large $\tan \beta$, the $m_{A}^{2}$ mass is almost determined by the $M_{1 / 2}^{2}$ contribution while a relatively large $m_{0}$ affects the Higgs mass only marginally. On the other hand, the allowed values for the trilinear parameter $A_{0}$ are also constrained by the requirement of absence of charge and colour breaking minima and it turns out typically that $-3<A_{0} / m_{0}<3$. In the analysis of the flavour physics observables the value of $A_{t}$ (together with the value of $\mu$ ) plays a particularly important role. The RGE equation for $A_{t}$ is:

$$
16 \pi^{2} \frac{d}{d t} A_{t} \simeq 12 A_{t} y_{t}^{2}-\frac{32}{3} g_{3}^{2} M_{3}
$$

where $t=\log \left(Q / M_{\mathrm{GUT}}\right)$. The LO solution of Eq. (14) provides the approximate result $A_{t}\left(m_{t}\right) \simeq 0.25 A_{t}(0)-2 M_{1 / 2}$. A relevant observation for the following discussions is that it is always possible to get small $\left|A_{t}\left(m_{t}\right)\right|$ values by opportunely selecting $A_{t}(0)$ and $M_{1 / 2}$. In summary the above qualitative considerations clearly show that we can have relatively small heavy Higgs masses in the CMSSM with large $\tan \beta$, small values of $M_{1 / 2}$ and relatively large values of $m_{0}$. This is the region of CMSSM parameter space that we will explore numerically in detail below.

Similarly, the NUHM MSSM is a simple extension of the CMSSM where the initial values of the Higgs masses, $m_{H_{d,}}$ and $m_{H_{\mu, 0}}$ are different from the rest of the sfermion masses, $m_{0}$ at the mediation scale [29, 30, 31, 32, 33, 34, 35, 36, 37, 38]. In this model we can expect that the additional freedom of varying the initial values of $m_{H_{d, 0}}$ and $m_{H_{u, 0}}$ can help to reduce $m_{A}^{2}$. In NUHM models, Eq. (13) is changed to [30]

$$
m_{H_{u}}^{2}\left(m_{t}\right) \simeq-0.75 m_{0}^{2}+0.63 m_{H_{u, 0}}^{2}-2.7 M_{1 / 2}^{2}+0.4 A_{0} M_{1 / 2}-0.1 A_{0}^{2}
$$

Similarly to the CMSSM case, the value of $m_{H_{d}}^{2}\left(m_{t}\right)$ depends strongly on $\tan \beta$. However, given that the bottom and tau Yukawa couplings are smaller than the top Yukawa up to values of $\tan \beta \gtrsim 50$, it is clear that we can expect the coefficient of $m_{H_{d, 0}}$ to be positive. 

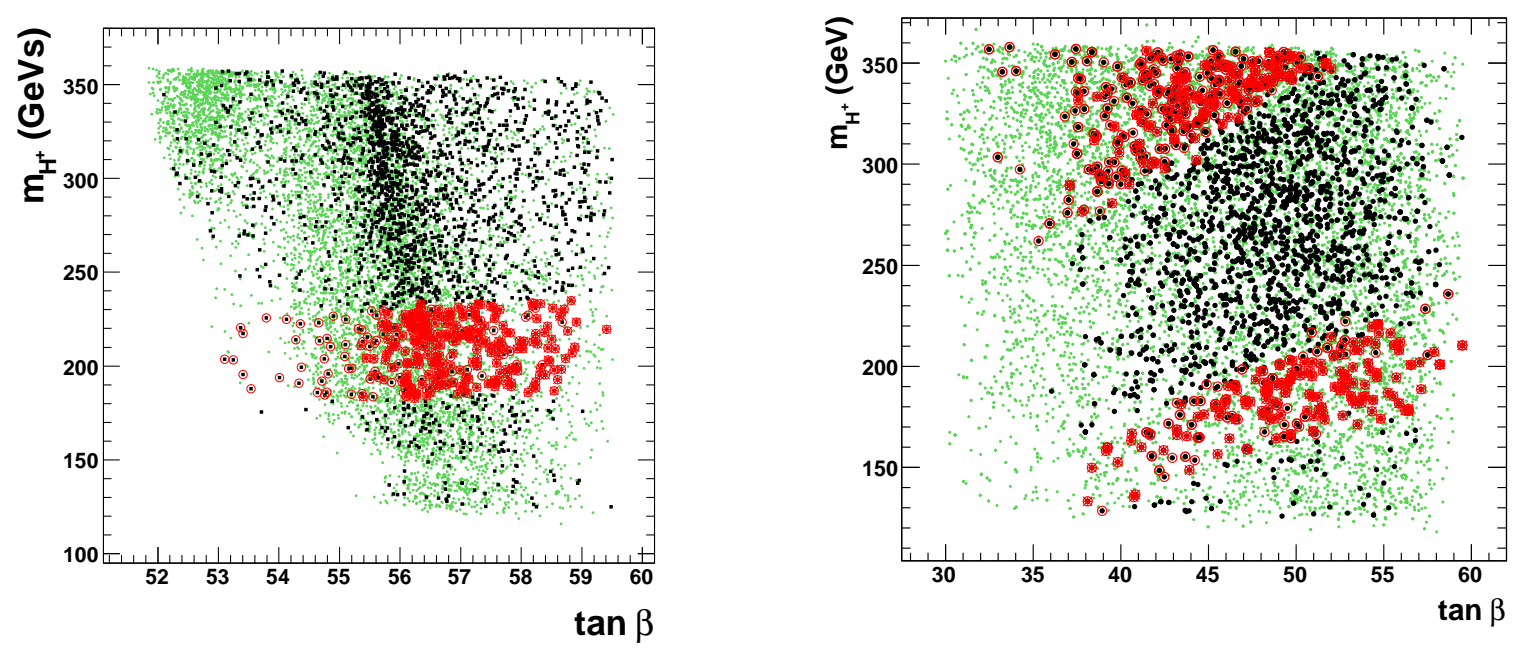

FIG. 1: Light values of $m_{H^{+}}$as a function of $\tan \beta$. In the CMSSM (left) we scan on $M_{1 / 2} \leq 600$ $\mathrm{GeV}, 900<m_{0}<2500 \mathrm{GeV}, \tan \beta>30$ and $-3 m_{0}<A_{0}<3 m_{0}$. In the NUHM (right), in addition we allow $0.75<m_{H_{u}} / m_{0}, m_{H_{d}} / m_{0}<1.25$. Green (light grey) points satisfy all direct bounds on scalar and gaugino masses. Black points satisfy also the main indirect constraints and $\Omega_{\chi}<0.14$ as explained in the text and red circles are points that in addition predict a $\operatorname{BR}(B \rightarrow \tau \nu)$ within the experimental range. Crossed red circles satisfy $0.08<\Omega_{\chi}<0.14$.

Then replacing these values in Eq. (9), we can see that the contribution of $m_{H_{u, 0}}$ to $m_{A}^{2}$ is negative while the contribution of $m_{H_{d, 0}}$ is positive (see for instance Table III in [30]). Therefore we can reduce $m_{A}^{2}$ in the NUHM with respect to the CMSSM for $m_{H_{d}}<m_{H_{u}}$.

Our numerical analysis below is done using RGE at two loop order [39] taking into account the complete flavour structure and the masses calculated at one-loop order [40] using SPheno [41]. In particular for the $\mu$ parameter and the neutral Higgs masses two-loop corrections are added [42]. We always impose the direct constraints on sfermion, gaugino and chargino masses from LEP and Tevatron [43]. In Figure 1 we show the values of $m_{H^{+}}$as a function of $\tan \beta$ for $\tan \beta>30$ in a scatter plot with $M_{1 / 2} \leq 600 \mathrm{GeV}$ and $900<m_{0}<2500 \mathrm{GeV}$ in the CMSSM and NUHM. All the points in these figures, including green points satisfy all direct bounds on scalar and gaugino masses. Ignoring indirect constraints for the moment, the most interesting feature here is the strong dependence of the mass with $\tan \beta$. We see that, indeed as discussed above, we can obtain charged Higgs masses below $200 \mathrm{GeV}$ in the 


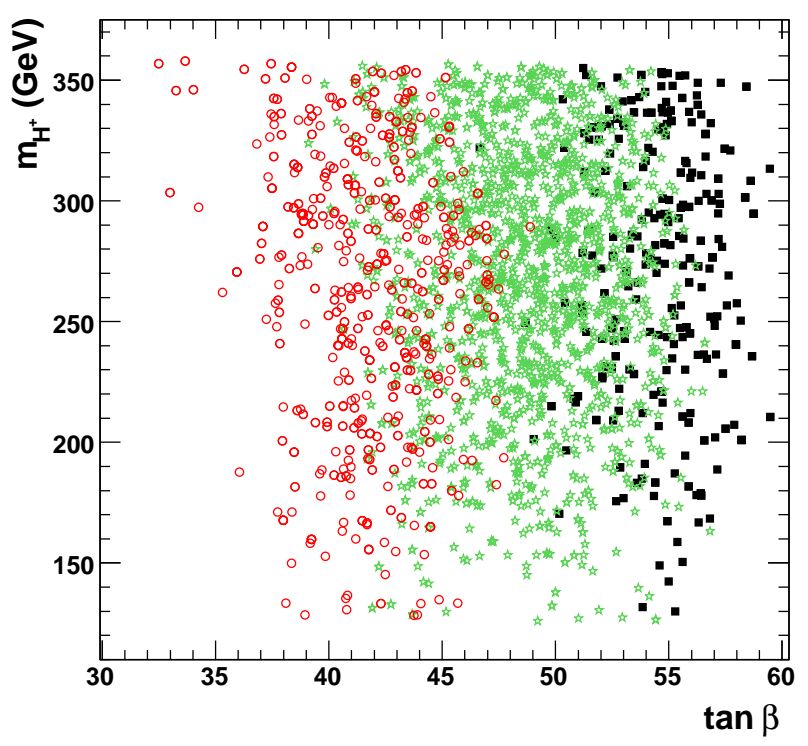

FIG. 2: Values of $m_{H^{+}}$as a function of $\tan \beta$ in the presence of non-universality at $M_{\mathrm{GUT}}$. Black squares correspond to points where $\left(m_{H_{u}}-m_{H_{d}}\right) / m_{0} \leq 0$, green (light grey) stars correspond to points with $0 \leq\left(m_{H_{u}}-m_{H_{d}}\right) / m_{0} \leq 0.3$ and red open circles to points where $0.3 \leq\left(m_{H_{u}}-m_{H_{d}}\right) / m_{0}$. All these points satisfy all direct and indirect bounds with the exception of $\operatorname{BR}(B \rightarrow \tau \nu)$. The upper bound on the dark matter abundance is also imposed.

CMSSM only for very large values of $\tan \beta, \tan \beta \geq 53$. Therefore, before imposing the dark matter and indirect constraints, it is possible to obtain charged Higgs masses below $200 \mathrm{GeV}$ in the CMSSM for $\mu>0, \tan \beta \geq 54, m_{0} \geq 900 \mathrm{GeV}$ and $M_{1 / 2} \leq 400 \mathrm{GeV}$. In the NUHM case, we have allowed a small departure from universality for the Higgs masses that can be $25 \%$ lighter or heavier that the common soft-mass $m_{0}$ at the GUT scale. In Figure 2 we can see the effect of a small non-universality in the GUT Higgs masses in the mass of the charged Higgs at $M_{W}$. As expected, the difference in the initial values of Higgs masses has a strong impact on the mass of the charged Higgs although we allow only a $25 \%$ departure for $m_{H_{u}}$ and $m_{H_{d}}$ from $m_{0}$. This becomes more pronounced if we allow for a larger breaking of universality. In the right-hand side plot of figure 1 we see that this small departure from universality is enough to obtain charged Higgs masses smaller than $200 \mathrm{GeV}$ for values of $\tan \beta$ as low as 30 before imposing the indirect constraints. 
Next, we must check that in this region of the parameter space it is possible to satisfy all the indirect constraints, specially those arising from processes enhanced by powers of $\tan \beta$, namely $B R(B \rightarrow \tau \nu), B R\left(B \rightarrow X_{s} \gamma\right), B_{s} \rightarrow \mu^{+} \mu^{-}$and the muon anomalous magnetic moment $(g-2)$. The behaviour of these observables in the large $\tan \beta$ regime was discussed in detail in Refs. [22, 23, 44, 45, 46, 47, 48, 49, 50, 51, 52, 53, 54, 55], ${ }^{4}$ and here we only summarize the main features.

On general grounds, the simultaneous requirement of a light $M_{A}$ and large $\tan \beta$ values strongly enhances Higgs mediated FCNC effects. Thus, very special conditions are necessary in order to satisfy all the phenomenological constraints, with special attention to the $B_{s} \rightarrow$ $\mu^{+} \mu^{-}$decay.

The decay $B \rightarrow X_{s} \gamma$ receives the dominant contributions from the $\mathrm{W}$-boson, the charged Higgs and the chargino diagrams. Gluino and neutralino contributions depend on radiatively generated Mass Insertions through the RG evolution, of order $c V_{t b} V_{t s}$, with $c$ a loop factor. Hence gluino and neutralino are subdominant with respect to the previous ones that do not have this additional loop suppression. However all contributions at one-loop order have been included in our numerical analysis below.

In the numerical analysis, we have imposed the allowed range for $B R\left(B \rightarrow X_{s} \gamma\right)$ as reported in Eq. (5) and we have evaluated $B R\left(B \rightarrow X_{s} \gamma\right)$ including the SM effects at the NNLO and the NP contributions at the LO. The charged Higgs contribution has always the same sign as the SM contribution. This contribution depends basically on the charged Higgs mass and it depends mildly on $\tan \beta$ through the threshold corrections to the bottom Yukawa coupling. Even though in the MSSM these threshold corrections reduce the size of the charged Higgs contribution (for $\mu>0$ ) compared to the 2HDM case, a charged Higgs of about $200 \mathrm{GeV}$ already saturates the allowed range for $B R\left(B \rightarrow X_{s} \gamma\right)$. Therefore, there is no space left for a chargino contribution with the same sign as the SM one. The relative sign between the chargino and the SM amplitudes is given by $\operatorname{sign}\left(A_{t} \mu\right)$. In the MSSM, except for very large $A_{0}>8 M_{1 / 2}$, we have always that $A_{t}\left(M_{W}\right)<0$. Under these conditions, a

\footnotetext{
${ }^{4}$ However, these analyses do not include the Higgs mediated FCNC contributions pointed out in Ref. [58]. As shown in Ref. [58], the renormalization of both $\tan \beta$ and the Higgs masses may lead to sizable effects for $\Delta M_{B_{s, d}}$ in the narrow region where $M_{A} \lesssim 160 \mathrm{GeV}$. Although this is exactly the region relevant for our analysis, we have checked numerically that the inclusion of these new effects do not lead to any further constraints on our scenario.
} 
$M_{A} \leq 200 \mathrm{GeV}$ forces to choose $\mu>0$ in order to get the necessary destructive interference between charged Higgs and chargino contributions to $B \rightarrow X_{s} \gamma$. Moreover, the chargino amplitude, $A_{\tilde{\chi}^{-}}$, is proportional to $A_{\tilde{\chi}^{-}} \propto\left[\mu A_{t} / m_{\tilde{q}}^{4}\right] \times \tan \beta$. Thus to keep $A_{\tilde{\chi}^{-}}$under control with very large values of $\tan \beta$, we need large sfermion masses and small $A_{t}$ and $\mu$. In fact this is precisely the situation we find in our numerical analysis, where all the squark masses are above one $\mathrm{TeV}$ and $\mu$, related to $m_{A}$, is relatively small.

The value of $A_{0}$ is scanned in the region $-3<A_{0} / m_{0}<3$ while we consider $M_{1 / 2} \leq 600$ $\mathrm{GeV}$. If we remember that $A_{t}\left(m_{t}\right) \simeq 0.25 A_{0}-2 M_{1 / 2}$, it is relatively easy to find small values for $A_{t}\left(m_{t}\right)$ when $A_{0}>0$ and for large $m_{0}$ compared to $M_{1 / 2}$, as it happens in the scenario we are considering.

Likewise, it is easy to find the SUSY contributions to the muon anomalous magnetic moment of the required size to explain its discrepancy with the SM expectation $\Delta a_{\mu}=$ $a_{\mu}^{\exp }-a_{\mu}^{\mathrm{SM}} \approx(3 \pm 1) \times 10^{-9}[54$, 56, 57]. This discrepancy can be accommodated only with a positive $\mu$ sign, in agreement with the $b \rightarrow s \gamma$ requirements. The main SUSY contribution to $a_{\mu}^{\mathrm{MSSM}}$ is provided by the loop exchange of charginos and sneutrinos. The basic features of the supersymmetric contribution to $a_{\mu}$ are correctly reproduced by the following approximate expression:

$$
\frac{a_{\mu}^{\mathrm{MSSM}}}{1 \times 10^{-9}} \approx 1.5\left(\frac{\tan \beta}{50}\right)\left(\frac{1000 \mathrm{GeV}}{m_{\tilde{\nu}}}\right)^{2},
$$

which provides a good approximation to the full one-loop result [59] when the chargino masses are substantially lighter then the slepton masses, as it happens in our case.

The SUSY contributions to $\operatorname{BR}\left(B_{s} \rightarrow \mu^{+} \mu^{-}\right)$can be summarized by the following approximate formula

$$
\operatorname{BR}\left(B_{s} \rightarrow \mu^{+} \mu^{-}\right) \simeq \frac{4 \times 10^{-8}}{\left[1+0.5 \times \frac{\tan \beta}{50}\right]^{4}}\left[\frac{\tan \beta}{50}\right]^{6}\left(\frac{160 \mathrm{GeV}}{M_{A}}\right)^{4}\left(\frac{\epsilon_{Y}}{4 \times 10^{-4}}\right)^{2}
$$

where $\epsilon_{Y}$ is defined through the flavor violating Yukawa interactions

$$
\mathcal{L}_{A}=\frac{i g_{2}}{2 M_{W}} m_{b} \frac{\epsilon_{Y} V_{t s} \tan _{\beta}^{2}}{\left(1+\epsilon_{0} \tan _{\beta}\right)^{2}} \bar{b}_{R} s_{L} A+\text { h.c. },
$$

It receives contributions both from charginos and gluinos ${ }^{5}$ thus, we can write $\epsilon_{Y}=\epsilon_{Y}^{\tilde{\chi}^{-}}+\epsilon_{Y}^{\tilde{g}}$

\footnotetext{
${ }^{5}$ Notice even though $\left(\delta_{L L}^{d}\right)_{23} / V_{t s}=O(0.1)$, gluino contributions to the effective $H_{u} b s$ vertex do not decouple and these contributions can play and important role when chargino contributions are reduced through a small $A_{t}$.
} 
with

$$
\epsilon_{Y}^{\tilde{\chi}^{-}} \simeq-\frac{1}{16 \pi^{2}} \frac{A_{t}}{\mu} H_{2}\left(y_{u_{R}}, y_{u_{L}}\right), \quad \epsilon_{Y}^{\tilde{g}} \simeq-\frac{2 \alpha_{3}}{3 \pi} \frac{\mu}{M_{\tilde{g}}} H_{3}\left(x_{d_{R}}, x_{d_{R}}, x_{d_{L}}\right) \frac{\left(\delta_{L L}^{d}\right)_{23}}{V_{t s}}
$$

with $y_{q_{R, L}}=M_{\tilde{q}_{L, R}}^{2} /|\mu|^{2}, x_{q_{R, L}}=M_{\tilde{q}_{L, R}}^{2} / M_{\tilde{g}}^{2}$, $\left(\delta_{L L}^{d}\right)_{23}$ the left-handed squark mass insertion at the electroweak scale and the loop functions are such that $H_{2}(1,1)=-1 / 2, H_{3}(1,1,1)=1 / 6$.

From Eq. (17), if $M_{A}=160 \mathrm{GeV}$ and $\tan \beta=50$, we can saturate the present experimental upper bound on $B R\left(B_{s} \rightarrow \mu^{+} \mu^{-}\right)$when $\epsilon_{Y} \simeq 4 \times 10^{-4}$. On the other hand, in the limit of all the SUSY masses and $A_{t}$ equal, the pure chargino contribution $\epsilon_{Y}^{\tilde{\chi}^{-}} \sim 3 \times 10^{-3}$. However, as discussed above, $A_{t}<0$ in most of the parameter space of SUGRA models so that, irrespective to the $\mu \operatorname{sign}, \operatorname{sgn}\left(\epsilon_{Y}^{\tilde{g}} / \epsilon_{Y}^{\tilde{\chi}^{-}}\right)=-1$ is unambiguously predicted when the sign of $\left(\delta_{L L}^{d}\right)_{23}$ is negative. We remind the reader that, even assuming a flavor blind soft sector at the GUT scale, running effects from $M_{G U T}$ down to the electroweak scale generate off-diagonal entries in the squark mass matrix as both type of Yukawa couplings, $Y_{u}$ and $Y_{d}$, contribute. Then we have $\left(\delta_{L L}^{d}\right)_{23} \simeq c \times V_{t s}$ with $c<0$ and typically $O(0.1)$ although it can be even close to 1 in special regions. Therefore cancellations between chargino and gluino contributions to $\epsilon_{Y}$ can be important when both contributions have similar sizes. $B R\left(B_{s} \rightarrow \mu \mu\right)$ is also reduced when both $\epsilon_{Y}^{\tilde{g}}$ and $\epsilon_{Y}^{\tilde{\chi}^{-}}$are small, i.e. when $\left(\delta_{L L}^{d}\right)_{23}$ and $A_{t}$ respectively are small. Given that $A_{t} \simeq 0.15 A_{0}-2 M_{1 / 2}$ (where the $M_{1 / 2}$ contributions are RGE induced and $\left.-3 \leq A_{0} / m_{0} \leq 3\right)$, it is clear that we can lower $A_{t}$ for large and positive $A_{0}$ values and moderate/small $M_{1 / 2}$; in this same region, the coefficient $c$ in $\left(\delta_{L L}^{d}\right)_{23}$ is large, and this makes the cancellation mechanism more effective. In the region of parameter space we explore, this is exactly the situation: we have both $m_{0}$ and $A_{0}$ large and $M_{1 / 2}$ small. Then $A_{t}$ (and also $\mu$ ) are relatively small when compared to the heavy sfermion masses. The arguments of the loop functions, specially $y_{q_{R, L}}$, are large and then $H_{2}(x>>1, y=x) \simeq-1 / x$ also reducing the chargino contribution.

Finally let us consider the $B \rightarrow \tau \nu$ decay. As shown in Eq. ([6), the ratio between the experimentally measured branching ratio and the $\mathrm{SM}$ expectation is given by $R_{B \tau \nu}^{\exp }=$ $1.07 \pm 0.42$. On the other hand, in SUSY, the charged-Higgs exchange contribution is [21, 22, 23, 24$]$

$$
R_{B \tau \nu}=\frac{\mathcal{B}^{\mathrm{SUSY}}\left(B_{u} \rightarrow \tau \nu\right)}{\mathcal{B}^{\mathrm{SM}}\left(B_{u} \rightarrow \tau \nu\right)}=\left[1-\left(\frac{m_{B}^{2}}{m_{H^{ \pm}}^{2}}\right) \frac{\tan ^{2} \beta}{\left(1+\epsilon_{0} \tan \beta\right)}\right]^{2},
$$

where non-holomorphic corrections to the down-type Yukawa coupling have been included. As evident from Eq. (20), $B_{u} \rightarrow \tau \nu$ represents a very powerful probe of the scenario we are 
exploring [21, 22, 23]. In contrast to $B_{s} \rightarrow \mu^{+} \mu^{-}, B_{u} \rightarrow \tau \nu$ is a tree-level process, thus, in this last case, there is no way to reduce the size of the NP contribution when $\tan \beta$ is large and the heavy Higgs is light. In fact, as we show below, we find that charged Higgs masses below $200 \mathrm{GeV}$ are only possible when the observed branching ration is obtained through a SUSY contribution twice the SM one (with opposite sign).

Similarly, charged scalar currents mediated by the charged Higgs affect also the process $K \rightarrow l \nu$ with $l=e, \mu$. The new physics effect in the ratio $R_{K \mu \nu}=B^{\mathrm{SUSY}}(K \rightarrow$ $\mu \nu) / B^{\mathrm{SM}}(K \rightarrow \mu \nu)$ would be obtained from Eq. (201) with the replacement $m_{B}^{2} \rightarrow m_{K}^{2}$. Although the charged Higgs contributions are now suppressed by a factor $m_{K}^{2} / m_{B}^{2} \simeq 1 / 100$, this is well compensated by the excellent experimental resolution [60, 61] and the good theoretical control. However, given that these new physics effects are at the $\%$ level, we would need a theoretical prediction for the SM contribution at the same level to use this decay as an effective constraint. We would then need an independent determination both of $f_{K}$ (possibly from lattice QCD) and $V_{u s}$. At present unquenched lattice calculations of $f_{K}$ are not well established and precise enough. The above argument for $K \rightarrow l \nu$ does not apply to $B \rightarrow \ell \nu$. In fact, even if the $f_{B}$ and $V_{u b}$ uncertainties are much larger that the $f_{K}$ and $V_{u s}$ ones, they cannot hide in any way the huge NP effects in $B \rightarrow \ell \nu$ arising in our scenario. Therefore, although it may play an important role in the future, we do not include the constraints from $K \rightarrow l \nu$ in the following.

In Fig. 11we can see the effect of these indirect constraints. Here, green (light grey) points satisfy only direct bounds while black points satisfy also the constraints from $\operatorname{BR}\left(B \rightarrow X_{s} \gamma\right)$, $\operatorname{BR}\left(B_{s} \rightarrow \mu^{+} \mu^{-}\right)$, the anomalous magnetic moment of the muon $(g-2)_{\mu}$ and the upper limit on the dark matter abundance $\Omega_{\chi}<0.14$. Both in the CMSSM and the NUHM we see that it is rather easy to satisfy these indirect constraints due to the relatively heavy sfermions and small $\mu$ and $A_{t}$. As expected, the main constraint here comes from the process $B_{u} \rightarrow \tau \nu$ that corresponds to the red circles in this figure. In the case of the CMSSM, we are bound to values of $\tan \beta>53$ for $m_{H^{+}}<200 \mathrm{GeV}$. Therefore, $B_{u} \rightarrow \tau \nu$ sets the lowest allowed value of $m_{H^{+}}$to $180 \mathrm{GeV}$. We have to emphasize again the importance of this tree-level constraint in this scenario. In the absence of this constraint, all black points would be allowed and hence charged Higgs masses as low as $120 \mathrm{GeV}$ would be possible in the CMSSM.

In the case of the NUHM, smaller values of $\tan \beta$ can still produce light charged Higgs masses as seen in Fig. 2. In the plot on the right-hand side of Fig. 1 we can clearly see the 

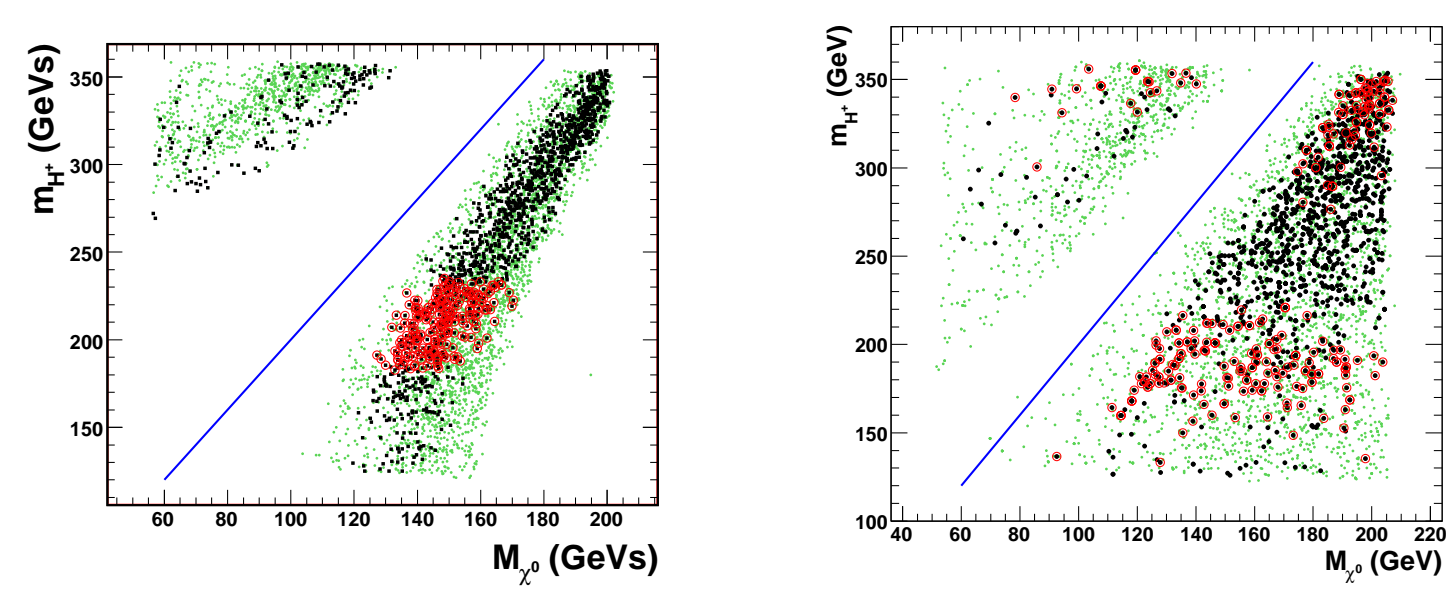

FIG. 3: Charged Higgs and lightest neutralino mass for the same points as Figure 1 both in the CMSSM (left) and in the NUHM (right). Green (light grey) points satisfy all direct bounds on scalar and gaugino masses and the dark matter constraint $0.08<\Omega_{\chi}<0.14$. Black points satisfy also the main indirect constraints as explained in the text. Red circles are points that in addition predict a $\mathrm{BR}(B \rightarrow \tau \nu)$ within the experimental range.

dependence of this decay on $\tan \beta / m_{H^{+}}$. Here it is possible to obtain charged Higgs masses as low as $120 \mathrm{GeV}$ for $\tan \beta=40$. Also in this plot we can see that without this cancellation of the SM contribution, the lower bound on the charged Higgs mass would be $350 \mathrm{GeV}$ for values of $\tan \beta=50$. That is why these points are not visible in the CMSSM plot.

Finally in Figure 3 we analyze the dark matter constraints on these points. The requirement of a correct dark matter abundance sets important restrictions on the allowed parameter space [33, 34, 35, 36, 62, 63, 64]. However, in this region of large $m_{0}$ and small $M_{1 / 2}$ this constraint is relatively easy to satisfy. We can see that both in the CMSSM and the NUHM all our points cluster in the funnel region on both sides of the line $m_{H^{+}}=2 m_{\chi}$. In fact in the NUHM we can see that there are points (not allowed by indirect constraints but satisfying the dark matter bound) much closer than expected to this central line. This is possible due to the fact that the annihilation cross section is proportional to $\tan ^{2} \beta$ and for lower values of $\tan \beta$ the allowed region is much closer to the resonance. Therefore we conclude that these points correspond basically to the funnel region, although significant contributions from other annihilation processes also occur. 
To conclude this section, we would like to comment on the differences between our analysis and the analysis presented in Ref. [3]. While on general grounds we agree with the conclusion of Ref. [3], there are some relevant differences that we would like to emphasize. First, the authors of Ref. [3] state that it is not possible in the CMSSM to find a light pseudoscalar reproducing the small CDF discrepancy consistently with all the constraints. In contrast, we have shown that a CMSSM can still accommodate such a scenario. Moreover, the authors

of Ref. [3] also claim that there exists a lower bound for $B R\left(B_{s} \rightarrow \mu \mu\right)$ of $B R\left(B_{s} \rightarrow \mu \mu\right)>$ $2 \times 10^{-8}$ in the region compatible with all the constraints. We disagree with this statement and we find no lower bound for $B R\left(B_{s} \rightarrow \mu \mu\right)$. Finally, in contrast to Ref. [3], we emphasize the fundamental impact of $B \rightarrow \tau^{+} \nu^{-}$. As we have discussed in detail, $B \rightarrow \tau^{+} \nu^{-}$represents probably the most important constraint/probe of this scenario.

\section{B. Other mediation mechanisms}

In gauge-mediated SUSY breaking scenarios [65], the SUSY breaking is transmitted to the MSSM sector through gauge interactions. In the minimal gauge-mediation (MGM) model, the messenger fields get fermionic masses $M_{M}=\lambda\langle S\rangle$ and scalar masses $m^{2}=$ $|\lambda\langle S\rangle|^{2} \pm\left|\lambda\left\langle F_{S}\right\rangle\right|$ through their Yukawa couplings to a singlet field $S$. Supersymmetry breaking is then transmitted to the MSSM gauginos and scalars through one-loop and twoloop diagrams respectively and we obtain:

$$
\begin{aligned}
& \bar{M}_{a}=N \frac{\bar{\alpha}_{a}}{4 \pi} \Lambda g(x) \equiv \widehat{M}_{a} g(x) \\
& \bar{m}_{\widetilde{\alpha}}^{2}=2 \Lambda^{2} N\left[C_{3}\left(\frac{\bar{\alpha}_{3}}{4 \pi}\right)^{2}+C_{2}\left(\frac{\bar{\alpha}_{2}}{4 \pi}\right)^{2}+\frac{3}{5} Y^{2}\left(\frac{\bar{\alpha}_{1}}{4 \pi}\right)^{2}\right] f(x) .
\end{aligned}
$$

These masses are fixed in terms of the overall scale parameter $\Lambda \equiv\left\langle F_{S}\right\rangle /\langle S\rangle$ and depend only very mildly on the mass ratios $x=\Lambda / M_{M}$. $C_{3}$ equals $4 / 3$ for squarks and 0 for sleptons, $C_{2}$ equals $3 / 4$ for $\mathrm{SU}(2)$ doublets and 0 for singlets, and $Y=Q-T_{3}$. In most of the parameter space that we analyze in the search of light charged-Higgs and large $\tan \beta$ we find that $f(x), g(x) \simeq 1$.

The allowed values for the pseudoscalar Higgs boson mass $m_{A}$ in MGM theories with radiative symmetry breaking can be found from the tree-level formula at the electroweak scale, Eq. (91). From Eqs. (21) and (22) we can relate $\bar{m}_{H_{u}}$ and $\bar{m}_{H_{d}}$ with gaugino masses at 
the messenger scale:

$$
\bar{m}_{H_{u}}^{2}=\bar{m}_{H_{d}}^{2} \simeq \frac{3}{2 N} \bar{M}_{2}+\frac{6}{5 N} \bar{M}_{1}
$$

After running to the electroweak scale, in the large $\tan \beta$ region these masses receive a large contribution proportional to $\bar{M}_{3}$ due to the stop and sbottom masses, while the dependence on $\bar{M}_{2}$ and $\bar{M}_{1}$ through $\bar{m}_{H_{u}}^{2}$ and $\bar{m}_{H_{d}}^{2}$ is reduced because of large top and bottom Yukawa couplings. In fact $m_{H_{u}}^{2}\left(M_{w}\right)$ and $m_{H_{d}}^{2}\left(M_{w}\right)$ are mainly determined by the values of $\bar{M}_{3}$. Therefore we can expect $m_{A}^{2} \simeq\left(C_{u}^{g}-C_{d}^{g}\right) M_{3}^{2}+\ldots$, with $C_{u}^{g}$ and $C_{d}^{g}$ being both $O(1)$ in the large $\tan \beta$ regime. In MGM the minimum value of $\bar{M}_{3}$ is fixed by the lower bound on the lightest stau mass:

$$
m_{\tilde{\tau}_{1}}^{2} \simeq m_{\tau_{R}}^{2}-m_{\tau} \mu \tan \beta \gtrsim(100 \mathrm{GeV})^{2}
$$

where from Eq. (22) we have, $m_{\tau_{R}}^{2} \simeq \frac{6}{5} \widehat{M}_{1}^{2}+s_{W}^{2} m_{Z}^{2}$. From here, we end up with $\widehat{M}_{1} \gtrsim$ $300 \mathrm{GeV}$ and thus $\widehat{M}_{3} \gtrsim 1350 \mathrm{GeV}$. Given that $m_{A}^{2} \simeq\left(C_{u}^{g}-C_{d}^{g}\right) \widehat{M}_{3}^{2}$, with both $C_{u}^{g}$ and $C_{d}^{g} O(1)$, a $m_{A} \sim 200 \mathrm{GeV}$ would require a strong cancellation between the two Yukawadependent coefficients at a level of $C_{u}^{g}-C_{d}^{g} \sim 10^{-2}$. This can be compared with the situation in the CMSSM where we can take $M_{1 / 2}$ of order $200 \mathrm{GeV}$ and we only need $C_{u}^{g}-C_{d}^{g} \sim 1$.

This estimate is confirmed by our numerical analysis, performed by means of a scanning over the MGM parameter space with SPheno [41]. In particular, we have not been able to find points with $m_{A}<300 \mathrm{GeV}$. Given that in the present analysis we are interested in scenarios allowing a light-heavy Higgs sector with $m_{A} \lesssim 200 \mathrm{GeV}$, the phenomenology of MGM models will be not analyzed.

In Anomaly Mediation the SUSY breaking is transmitted from the hidden sector by the the superconformal anomaly [66, 67]. All the soft-breaking parameters are determined in a renormalization group invariant way by a single parameter, the gravitino mass. The soft-breaking parameters are given by:

$$
M_{a}=\frac{1}{g_{a}} \beta_{a} m_{3 / 2}, \quad m_{i}^{2}=\frac{1}{2} \dot{\gamma}_{i} m_{3 / 2}^{2}, \quad A_{i}=\beta_{Y_{i}} m_{3 / 2},
$$

where $\beta_{a}$ and $\beta_{Y_{i}}$ are the beta functions of gauge and Yukawa couplings, $\gamma_{i}$ the anomalous dimension of the corresponding matter superfield and $m_{3 / 2}$ the gravitino mass. Unfortunately, pure anomaly mediation is not acceptable because it leads to tachyonic sleptons. The different approaches to solve this problem make the analysis highly model dependent. A simple solution to this problem maintaining also the renormalization group invariance is 
to add a (or several) Fayet-Iliopoulos D-term contribution(s) to the scalar masses [68, 69]. In this way the scalar masses in Eq. (25) are replaced by $m_{i}^{2}=\frac{1}{2} \dot{\gamma}_{i} m_{3 / 2}^{2}+m_{0}^{2} Y_{i}$, where $m_{0}$ is the D-term contribution and $Y_{i}$ the charge corresponding to the new broken $U(1)$ symmetry. The spectrum depends then on the charges of the SM particles under the new $U(1)$ groups. Although we do not make a full analysis, following Ref. [69], we can see that the requirement of $m_{A}>90 \mathrm{GeV}$ sets one of the limits on the allowed region of the parameter space. Therefore in these particular models it is relatively easy to have pseudoscalar masses below $200 \mathrm{GeV}$. However, a correct electroweak symmetry breaking is obtained only for values of $\tan \beta<27$ making most of the phenomenology and specially indirect searches less interesting [69]. In Ref. [57] a different solution to the tachyonic problem is analyzed with similar results. A complete analysis of more general anomaly mediation models is indeed interesting and will be discussed elsewhere.

\section{GENERIC SIGNALS OF THE LIGHT CHARGED-HIGGS SCENARIO}

As we have seen in the previous section, it is still possible to have a light charged Higgs both in CMSSM and in NUHM models consistent with all the phenomenological constraints. At this point we can ask what would be the signatures of this scenario. We will discus the possible signals both at high-energy colliders (LHC, Tevatron) and at low-energy flavour changing experiments.

\section{A. Direct searches at colliders}

The expected spectrum in the light charged-Higgs scenario is somewhat peculiar. In Table \ we present the allowed range of input parameters in the CMSSM for points with a charged-Higgs below $200 \mathrm{GeV}$ satisfying all direct constraints and indirect constraints and a dark matter abundance in the range $0.08<\Omega_{\chi}<0.14$. As we can see, the main features of this region of parameter space are $M_{1 / 2}<<m_{0}$ and $\tan \beta>55$. As a consequence, we can expect relatively light gauginos and heavy sfermions. This is confirmed in Table II where we show the obtained mass ranges with these input parameters. In this table we see that sfermions of the first two generations are roughly above $1 \mathrm{TeV}$. Only sfermions of the third generation can be relatively light due to the effect of the large Yukawa couplings. 


\begin{tabular}{||ll||}
\hline CMSSM parameter & Allowed range \\
\hline$m_{0}$ & $900-1400 \mathrm{GeV}$ \\
\hline$M_{1 / 2}$ & $320-440 \mathrm{GeV}$ \\
\hline$A_{0}$ & $350-1700 \mathrm{GeV}$ \\
\hline $\tan \beta$ & $55-60$ \\
\hline $\operatorname{sign}(\mu)$ & +1 \\
\hline
\end{tabular}

TABLE I: Input ranges in the CMSSM for points with $m_{A}<200 \mathrm{GeV}$ and satisfying direct, indirect and dark matter constraints.

\begin{tabular}{|c|c||c|c|}
\hline \hline & mass $(\mathrm{GeV})$ & & mass $(\mathrm{GeV})$ \\
\hline$\chi_{1}$ & $130-180$ & $\chi_{2}$ & $250-330$ \\
\hline$\chi_{3}$ & $430-540$ & $\chi_{4}$ & $450-550$ \\
\hline$\chi_{1}^{ \pm}$ & $250-330$ & $\chi_{2}^{ \pm}$ & $450-550$ \\
\hline$\tilde{g}$ & $820-1050$ & & \\
\hline$\tilde{t}_{1}$ & $780-1050$ & $\tilde{t}_{2}$ & $890-1170$ \\
\hline$\tilde{b}_{1}$ & $850-1150$ & $\tilde{b}_{2}$ & $930-1200$ \\
\hline$\tilde{u}_{R}$ & $1160-1550$ & $\tilde{u}_{L}$ & $1180-1560$ \\
\hline$\tilde{d}_{R}$ & $1150-1550$ & $\tilde{d}_{L}$ & $1170-1570$ \\
\hline$\tilde{\tau}_{1}$ & $320-860$ & $\tilde{\tau}_{2}$ & $720-1160$ \\
\hline$\tilde{e}_{R}$ & $900-1360$ & $\tilde{e}_{L}$ & $920-1380$ \\
\hline$\tilde{\nu}_{1}$ & $700-1160$ & $\tilde{\nu}_{3}$ & $920-1380$ \\
\hline$h$ & $112.4-115.6$ & $H$ & $165-200$ \\
\hline$A$ & $165-200$ & $H^{ \pm}$ & $180-210$ \\
\hline
\end{tabular}

TABLE II: Mass ranges in the CMSSM for the input parameters in Table I.

In the NUHM the allowed range of input parameters is shown in Table III. The main difference between the CMSSM and NUHM input parameters is the allowed range of $\tan \beta$ compatible with a light charged-Higgs below $200 \mathrm{GeV}$. In fact, values of $\tan \beta \gtrsim 40$ are allowed in the NUHM case. This implies that lower $M_{1 / 2}$ values with respect to the CMSSM case are now allowed. Lower $M_{1 / 2}$ implies lighter squarks and gauginos that increase the 


\begin{tabular}{||ll||}
\hline \hline CMSSM parameter & Allowed range \\
\hline$m_{0}$ & $760-1280 \mathrm{GeV}$ \\
\hline$m_{H_{d 0}}$ & $660-1380 \mathrm{GeV}$ \\
\hline$m_{H_{u} 0}$ & $820-1520 \mathrm{GeV}$ \\
\hline$M_{1 / 2}$ & $180-480 \mathrm{GeV}$ \\
\hline$A_{0}$ & $400-2150 \mathrm{GeV}$ \\
\hline $\tan \beta$ & $39-60$ \\
\hline $\operatorname{sign}(\mu)$ & +1 \\
\hline
\end{tabular}

TABLE III: Input ranges in the NUHM for points with $m_{A}<200 \mathrm{GeV}$ and satisfying direct, indirect and dark matter constraints.

SUSY contribution to $B R(B \rightarrow s \gamma)$. However in the NUHM scenario, this can be compensated by selecting lower $\tan \beta$ values. The particle mass ranges in NUHM models are shown in Table IV. The comments made for the CMSSM apply also here. Notice that the CMSSM is a particular case of the NUHM, so all the allowed points in the CMSSM are also allowed in the NUHM.

Let us first discus the Higgs sector in this scenario. As can be seen in Tables [I] and IV] both the CMSSM and NUHM predict the lightest Higgs boson in the range $m_{h} \simeq 112-116$ $\mathrm{GeV}$. Notice that our scenario corresponds, by construction, to the non-decoupling regime of the MSSM. Hence the LEP bound on the SM Higgs mass of $114.4 \mathrm{GeV}$ does not apply in our case. In general values as low as $90 \mathrm{GeV}$ for the lightest Higgs mass are allowed in this regime [70]. The lower value for the lightest Higgs mass we obtain is due to the heavy squark masses and large $\tan \beta$ values. As we have discussed, we select the points in the parameter space in order to get charged Higgs masses $\lesssim 200 \mathrm{GeV}$. This opens the possibility of interesting experimental signatures at colliders. In particular, one of the more interesting possibilities would be to look for the top decay via $t \rightarrow H^{+} b$, which is allowed for $m_{t}>m_{H^{+}}+m_{b}$. Otherwise, when this decay is not allowed, charged-Higgs decays are also interesting. The charged Higgs boson decays mainly to $\bar{\tau} \nu$ or to $t b$ depending on $m_{H^{+}}-m_{t}-m_{b}$. In the left-hand side plot of Fig. 4 we show the $B R\left(t \rightarrow H^{+} b\right)$ as a function of the charged Higgs mass $m_{H^{+}}$imposing all the constraints on the SUSY spectrum from flavor and EWPO observables. We can see that branching ratios at the few per cent level 


\begin{tabular}{|c|c||c|c|}
\hline \hline & mass $(\mathrm{GeV})$ & & mass $(\mathrm{GeV})$ \\
\hline$\chi_{1}$ & $65-195$ & $\chi_{2}$ & $120-370$ \\
\hline$\chi_{3}$ & $160-640$ & $\chi_{4}$ & $240-640$ \\
\hline$\chi_{1}^{ \pm}$ & $110-370$ & $\chi_{2}^{ \pm}$ & $240-650$ \\
\hline$\tilde{g}$ & $480-1140$ & & \\
\hline$\tilde{t}_{1}$ & $710-970$ & $\tilde{t}_{2}$ & $870-1120$ \\
\hline$\tilde{b}_{1}$ & $840-1100$ & $\tilde{b}_{2}$ & $900-1210$ \\
\hline$\tilde{u}_{R}$ & $1080-1520$ & $\tilde{u}_{L}$ & $1080-1540$ \\
\hline$\tilde{d}_{R}$ & $1070-1520$ & $\tilde{d}_{L}$ & $1080-1540$ \\
\hline$\tilde{\tau}_{1}$ & $200-1060$ & $\tilde{\tau}_{2}$ & $620-1200$ \\
\hline$\tilde{e}_{R}$ & $780-1300$ & $\tilde{e}_{L}$ & $800-1310$ \\
\hline$\tilde{\nu}_{1}$ & $610-1190$ & $\tilde{\nu}_{3}$ & $800-1310$ \\
\hline$h$ & $112.4-115.6$ & $H$ & $128-200$ \\
\hline$A$ & $128-200$ & $H^{ \pm}$ & $148-210$ \\
\hline
\end{tabular}

TABLE IV: Mass ranges in the NUHM for the input parameters in Table III.

are possible. Given that the LHC will be a top factory producing $10^{7}$ top pairs already with $10 \mathrm{fb}^{-1}$, the $t \rightarrow \mathrm{H}^{+} \mathrm{b}$ process clearly represents a very clean signature of our scenario if $m_{t}>m_{H^{+}}+m_{b}$, which is only possible in the NUHM model. Remember that in the CMSSM $\tan \beta>53$ for $m_{H^{+}}<200 \mathrm{GeV}$ and then the constraints from $B_{u} \rightarrow \tau \nu$ forbid completely this possibility. Charged Higgs decays are also interesting in general. In the right-hand side plot of Fig. 4 we report the $B R\left(H^{+} \rightarrow t b\right)$ and $B R\left(H^{+} \rightarrow \tau \nu\right)$ 's as function of $m_{H^{+}}$. We note that $\left.\Gamma\left(H^{+} \rightarrow \mathrm{All}\right) \simeq \Gamma\left(\mathrm{H}^{+} \rightarrow \mathrm{tb}\right)+\Gamma\left(\mathrm{H}^{+} \rightarrow \tau \nu\right)\right)$ and that $B R\left(H^{+} \rightarrow t b\right)$ increase while increasing $m_{H^{+}}$as it is understandable by kinematical considerations. On the other hand, the $H^{+} \rightarrow \tau \nu$ decay mode starts being the dominant one when $m_{H^{+}} \leq 220 \mathrm{GeV}$.

As discussed above, our scenario predicts relatively light gaugino masses. In particular the gluino mass turns out to be usually lighter than the squarks. This has important phenomenological consequences at hadronic colliders. In fact from Tables II and IV we can see that the gluino is always lighter than squarks of the first two generations thus the decay $\tilde{g} \rightarrow q_{1,2} \tilde{q}_{1,2}$ is never allowed. In general this is not always true for squarks of the third 

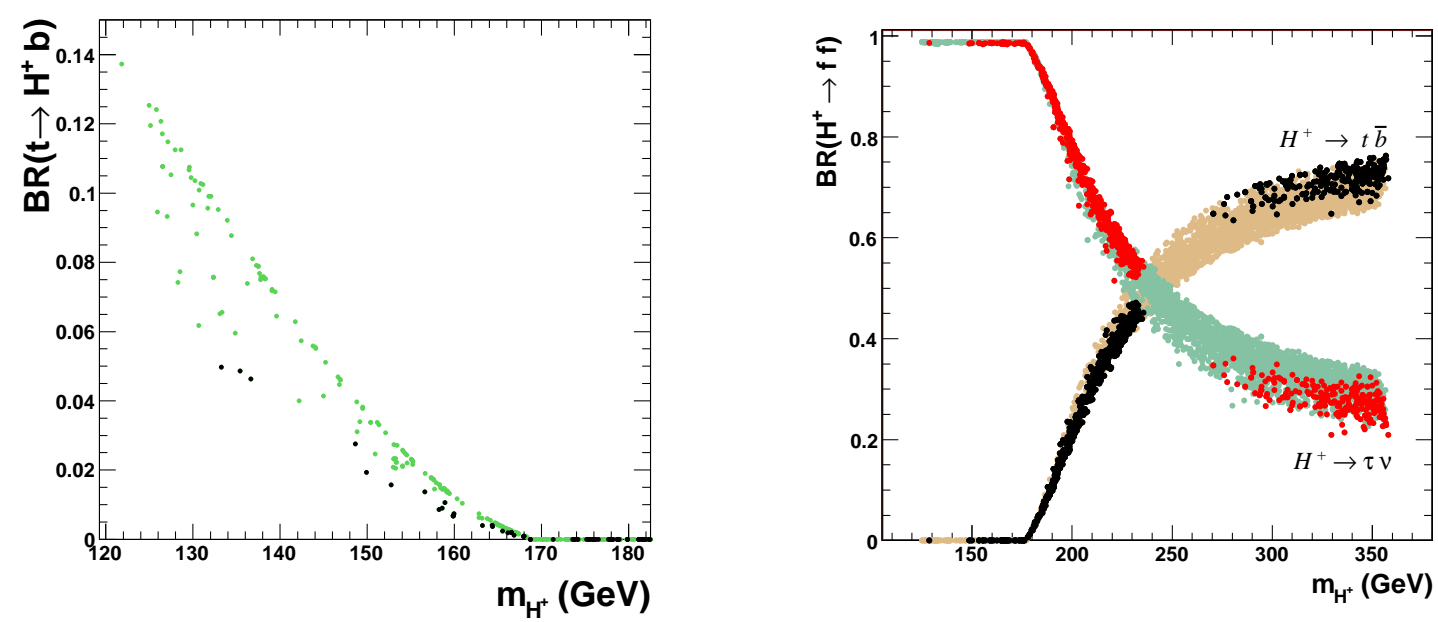

FIG. 4: Left: $B R\left(t \rightarrow H^{+} b\right)$ as a function of $m_{H^{+}}$. Right: $B R\left(H^{+} \rightarrow t b\right)$ and $B R\left(H^{+} \rightarrow \tau \nu\right)$ 's as function of $m_{H^{+}}$. In both plots, clear (green or orange) points satisfy all the constraints on the SUSY spectrum from flavor and EWPO observables with the exception of $B R\left(B_{u} \rightarrow \tau \nu\right)$. Dark (red or black) points satisfy in addition the constraints from $B R\left(B_{u} \rightarrow \tau \nu\right)$.

generation as can be seen in the tables. However, in our numerical analysis we find that the decay $\tilde{g} \rightarrow t \tilde{t}_{1}$ is never kinematically allowed. On the contrary, $\tilde{g} \rightarrow b \tilde{b}_{1}$ is possible for $\lesssim 10 \%$ of the allowed points. Notice that this fact is only due to the small bottom mass compared to the top mass. If none of these decays is kinematically allowed, gluino has either three body decays into two quarks and either a neutralino or a chargino or the loop induced two-body decay into $g \tilde{\chi}_{i}^{0}$. In the scenarios discussed above $\tan \beta$ is relatively large implying that final states containing quarks of the 3rd generations are strongly preferred [71]. The branching ratios of the final states $b \bar{b} \tilde{\chi}_{i}^{0}$ are enhanced compared to $t \bar{t} \tilde{\chi}_{i}^{0}$ due to obvious kinematical reasons. Therefore, independent of kinematics we expect in gluino decays an enhancement of final states containing b-quarks.

\section{B. Indirect FCNC searches}

The main features of our scenario are large $\tan \beta$ and light Higgs masses. Therefore we can expect sizeable SUSY contributions to $\tan \beta$-enhanced decays, specially in $B_{s} \rightarrow \mu^{+} \mu^{-}$ 
and $B \rightarrow \tau^{+} \nu^{-}$. Even though we find very large contributions to the above observables in most of the points of our numerical analysis, we must stress that it is not guaranteed that an experimental/theoretical improvement in these decays would find a departure from the SM expectations. In fact, in the $B_{s} \rightarrow \mu^{+} \mu^{-}$case we can always find regions where the smallness of $A_{t}$ and/or gluino-chargino cancellations can reduce SUSY contributions to the level of the SM. With respect to the $B \rightarrow \tau^{+} \nu^{-}$decay we stress that the light-Higgs scenario with large $\tan \beta$ values can accommodate the present experimental determination only when the SUSY contribution is roughly twice the SM one (note that the charged-Higgs contribution has always opposite sign compared to the SM one). Therefore, even if tuned, it is always possible to find a $\left(\tan \beta, m_{H^{+}}\right)$combination reproducing the SM prediction for this branching ratio. However we must emphasize that both decays are probably the most promising indirect channels to look for the light charged-Higgs scenario.

In addition to these hadronic observables, lepton flavour violating (LFV) transitions, as $\ell_{i} \rightarrow \ell_{j} \gamma$, are also very sensitive probes of the large $\tan \beta$ scenario. Unfortunately these decays require an additional source of LFV. However, LFV couplings naturally appear in the MSSM once we extend it to accommodate the non-vanishing neutrino masses and mixing angles by means of a supersymmetric seesaw mechanism [72, 73]. In this case, LFV entries in the slepton mass matrix $\left(m_{\tilde{L}}^{2}\right)_{i j}$ are radiatively induced [72]:

$$
\delta_{L L}^{i j}=\frac{\left(M_{\tilde{\ell}}^{2}\right)_{L_{i} L_{j}}}{\sqrt{\left(M_{\tilde{\ell}}^{2}\right)_{L_{i} L_{i}}\left(M_{\tilde{\ell}}^{2}\right)_{L_{j} L_{j}}}} \approx-\frac{\left(3+A_{0}^{2}\right)}{8 \pi^{2}} \log \left(\frac{M_{X}}{M_{R}}\right)\left(Y_{\nu}^{\dagger} Y_{\nu}\right)_{i j},
$$

where $Y_{\nu}$ are the neutrino Yukawa couplings (the potentially large sources of LFV) and $M_{X}$ and $M_{R}$ are the GUT and the heavy right handed neutrino masses, respectively. In our analysis, we consider a rather conservative situation where the mixing angles in the neutrino Yukawa matrix are small, CKM-like [74], and the largest neutrino Yukawa eigenvalue is $O(1)$ similarly to the top Yukawa.

In Fig. 5 on the left-(right-)hand side, we report the predictions of the CKM-like scenario for $\mu \rightarrow e(\tau \rightarrow \mu)$ transitions as a function of $\Delta a_{\mu}$ employing the ranges for the input parameters listed in Table I. We set $y_{\nu_{3}}=1, M_{X}=2 \times 10^{16} \mathrm{GeV}$ and $M_{R}=10^{15} \mathrm{GeV}$, as it would be obtained via the see-saw formula with a hierarchical light neutrino spectrum with $m_{\nu_{1}} \simeq 10^{-3} \mathrm{eV}$. Notice that in this figure we present the predictions for LFV processes in the CMSSM scenario; in fact, within the region of parameter space of our interest, the 

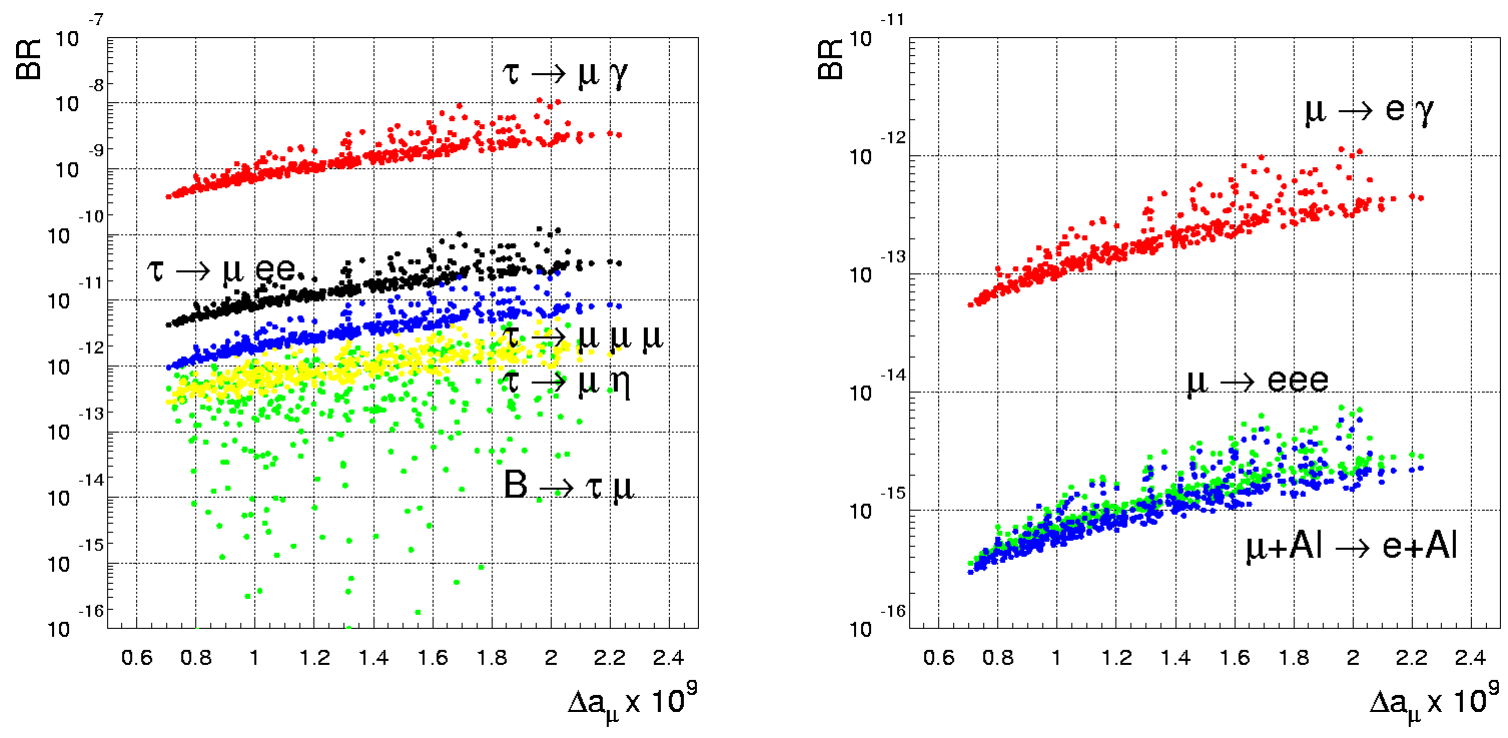

FIG. 5: Left (Right): Expectations for $\tau \rightarrow \mu(\mu \rightarrow e)$ transitions vs. $\Delta a_{\mu}=\left(g_{\mu}-g_{\mu}^{\mathrm{SM}}\right) / 2$ (remind that $\left.\Delta a_{\mu}=a_{\mu}^{\exp }-a_{\mu}^{\mathrm{SM}} \approx(3 \pm 1) \times 10^{-9}\right)$ assuming a CKM-like scenario. The plots have been obtained employing the ranges for the input parameters listed in Table \.

CMSSM and NUHM models (with RH neutrinos) have very similar predictions. Given that both $\ell_{i} \rightarrow \ell_{j} \gamma$ and $\Delta a_{\mu}=\left(g_{\mu}-g_{\mu}^{\mathrm{SM}}\right) / 2$ are generated by dipole operators, it is natural to expect that their amplitudes are closely connected [23, 75]. In particular, assuming a CMSSM spectrum, it is found that

$$
\mathcal{B}\left(\ell_{i} \rightarrow \ell_{j} \gamma\right) \approx\left[\frac{\Delta a_{\mu}}{30 \times 10^{-10}}\right]^{2} \times \begin{cases}10^{-12}\left|\frac{\delta_{L L}^{12}}{4 \times 10^{-5}}\right|^{2} & {[\mu \rightarrow e]} \\ 10^{-8}\left|\frac{\delta_{L L}^{23}}{6 \times 10^{-3}}\right|^{2} & {[\tau \rightarrow \mu]}\end{cases}
$$

where $\delta_{L L}^{i j}$ has been evaluated by means of Eq. (26) for $A_{0}=1$. As we can see, the correlation is not exactly a line as one would expect from Eq. (27), since i) the loop functions for the two processes are not identical, ii) while $B R\left(\ell_{i} \rightarrow \ell_{j} \gamma\right)$ strongly depends on $A_{0}$ through $\delta_{L L}^{i j}$ (see Eq. (26) $), \Delta a_{\mu}$ is almost insensitive to $A_{0}$. From Fig. 5 we see that, although model dependent, both $\mu \rightarrow e \gamma$ and $\tau \rightarrow \mu \gamma$ branching ratios could naturally reach the experimentally projected sensitivities in MEG and SuperB factories. This is specially true in the interesting region of the SUSY parameter space where also the $(g-2)_{\mu}$ anomaly, i.e. $\Delta a_{\mu}=a_{\mu}^{\exp }-a_{\mu}^{\mathrm{SM}} \approx(3 \pm 1) \times 10^{-9}$, can find a natural explanation.

Furthermore, we note that, although our scenario has a very light heavy Higgs sector 
$m_{A} \lesssim 200 \mathrm{GeV}$ and large $\tan \beta$ values, $\tan \beta \gtrsim 40$, Higgs mediated LFV transitions [76, 77, 78, 79, 80, 81] are not particularly enhanced, as could be expected. The reason for this is that Higgs mediated lepton flavour violating couplings are quite suppressed because of the large mass splitting between the gaugino and sfermion masses. In particular, as we can see from Fig. 5, both $B R(\tau \rightarrow \mu \eta)$ and $B R(B \rightarrow \tau \mu)$ (that are purely Higgs mediated processes [76]), never exceed the level of few $10^{-12}$. Higgs mediated contributions to $\tau \rightarrow \mu \mu \mu$ are completely subdominant compared to the dipole $\left(\tau \rightarrow \mu \gamma^{*}\right)$ effects.

As it concerns the $\mu \rightarrow e$ transitions, scalar current effects contribute quite sizably only to $B R(\mu+A l \rightarrow e+A l)$. However, for the parameter space relevant in our analysis, both $B R(\mu+A l \rightarrow e+A l)$ and $B R(\mu \rightarrow e e e)$ lie below the $10^{-14}$ level, well far from their current experimental resolutions.

Finally, the predictions for $\tau \rightarrow e$ transitions are simply obtained from those for the $\tau \rightarrow \mu$ transitions by $B R(\tau \rightarrow e X)=\left|V_{t d} / V_{t s}\right|^{2} B R(\tau \rightarrow e X)$ (with $X=\gamma, \eta, \mu \mu, e e$ ) and $B R(B \rightarrow \tau e)=\left|V_{t d} / V_{t s}\right|^{2} B R(B \rightarrow \tau \mu)$.

\section{CONCLUSIONS AND OUTLOOK}

In this paper we have investigated whether the possibility of having a charged-Higgs boson with a mass below $200 \mathrm{GeV}$ is still open. We have answered this question both in the context of a 2HDM and in the framework of the MSSM. In the 2HDM, the chargedHiggs mass is constrained to be above $295 \mathrm{GeV}$ by $\mathrm{BR}(b \rightarrow s \gamma)$ [15], although we have found that a pseudoscalar mass in the range $150-200 \mathrm{GeV}$ is still allowed. In the context of the MSSM we have seen that a light charged-Higgs below $200 \mathrm{GeV}$ is still possible both in the Constrained MSSM (CMSSM) and in a MSSM with non-universal Higgs masses. Light Higgs masses in these supersymmetric scenarios require always very large values of $\tan \beta$. These models, in the light $m_{H^{+}}$-large $\tan \beta$ region have to face strong restrictions from the $B \rightarrow \tau \nu$ decay, that turns out to be the strongest constraint of our scenario. In particular, in the CMSSM $\tan \beta$ is always larger than 50 when we want $m_{H^{+}}<200 \mathrm{GeV}$ and then the $B \rightarrow \tau \nu$ decay sets a strict lower limit of $180 \mathrm{GeV}$ for the charged-Higgs mass. This lower limit from $B \rightarrow \tau \nu$ is relaxed in NUHM models where we can obtain light charged Higgses with smaller values of $\tan \beta$. Moreover, we have analyzed the generic predictions of our light charged-Higgs scenario for hadronic colliders and indirect searches. Finally, we have 
addressed the question whether the above scenario can be tested through LFV processes. To this purpose, we have considered a rather conservative ansatz for the source of LFV, the so-called CKM-like [74] case, and we have evaluated the predictions for the most relevant low-energy LFV processes. Interestingly enough, both $\mu \rightarrow e \gamma$ and $\tau \rightarrow \mu \gamma$ branching ratios naturally reach the experimentally projected sensitivities in MEG and SuperB factories, specially in the region where the $(g-2)_{\mu}$ anomaly can find a natural explanation.

\section{Acknowledgments}

We thank M.Antonelli, U.Haisch, G.Isidori, J.Hisano, M. Carena and C. Wagner for useful discussions. G.B., P.P. and O.V. acknowledge partial support from the Spanish MCYT FPA2005-01678, Generalitat Valenciana under contract GV05/207 and the EU MRTN-CT2006-035482 (FLAVIAnet). W.P. is partially supported by the German Ministry of Education and Research (BMBF) under contract 05HT6WWA. E.L. is supported by the Department of Energy under Grant DE-AC02-76CH030000. E.L and P.P thank the Aspen Center for Physics, where part of this work was done. Fermilab is operated by Fermi Research Alliance, LLC under Contract No. DE-AC02-07CH11359 with the United States Department of Energy.

[1] CDF Collaboration, CDF note 8954, see : http://www-cdf.fnal.gov/physics/new/hdg/results/3b_susyhiggs_070803/ cdf8954_higgs3b_v10.pdf .

[2] D0 Collaboration, D0 Note 5503-CONF, see: http://www-d0.fnal.gov/Run2Physics/WWW/results/prelim/HIGGS/H23/H23.pdf.

[3] J. R. Ellis, S. Heinemeyer, K. A. Olive and G. Weiglein, Phys. Lett. B 653 (2007) 292 arXiv:0706.0977 [hep-ph]].

[4] D. Feldman, Z. Liu and P. Nath, arXiv:0711.4591 [hep-ph].

[5] J. F. Gunion, H. E. Haber, G. L. Kane and S. Dawson, “The Higgs Hunter's Guide” (AddisonWesley, New York, 1990).

[6] B. W. Lee, C. Quigg and H. B. Thacker, Phys. Rev. Lett. 38 (1977) 883. 
[7] S. Kanemura, T. Kubota and E. Takasugi, Phys. Lett. B 313 (1993) 155 arXiv:hep-ph/9303263.

[8] H. Huffel and G. Pocsik, Z. Phys. C 8 (1981) 13; J. Maalampi, J. Sirkka and I. Vilja, Phys. Lett. B 265 (1991) 371; A. G. Akeroyd, A. Arhrib and E. M. Naimi, Phys. Lett. B 490 (2000) 119 arXiv:hep-ph/0006035; I. F. Ginzburg and I. P. Ivanov, arXiv:hep-ph/0312374.

[9] M. E. Peskin and T. Takeuchi, Phys. Rev. D 46 (1992) 381.

[10] A. Wahab El Kaffas, P. Osland and O. Magne Ogreid, Phys. Rev. D 76 (2007) 095001 arXiv:0706.2997 [hep-ph]].

[11] N. G. Deshpande and E. Ma, Phys. Rev. D 18 (1978) 2574; S. Nie and M. Sher, Phys. Lett. B 449 (1999) 89 arXiv:hep-ph/9811234.

[12] H. E. Haber and A. Pomarol, Phys. Lett. B 302 (1993) 435 arXiv:hep-ph/9207267; A. Pomarol and R. Vega, Nucl. Phys. B 413 (1994) 3 arXiv:hep-ph/9305272.

[13] C. S. Lim, T. Inami and N. Sakai, Phys. Rev. D 29 (1984) 1488.

[14] E. Barberio et al. [Heavy Flavor Averaging Group (HFAG) Collaboration], arXiv:0704.3575 [hep-ex].

[15] M. Misiak and M. Steinhauser, Nucl. Phys. B 764 (2007) 62 arXiv:hep-ph/0609241.

[16] M. Misiak et al., Phys. Rev. Lett. 98 (2007) 022002 arXiv:hep-ph/0609232.

[17] T. Hurth, E. Lunghi and W. Porod, Nucl. Phys. B 704, 56 (2005) arXiv:hep-ph/0312260.

[18] E. Lunghi and J. Matias, JHEP 0704, 058 (2007) arXiv:hep-ph/0612166.

[19] B. Aubert et al. [BABAR Collaboration], Phys. Rev. D 76 (2007) 052002 arXiv:0708.2260 [hep-ex]].

[20] K. Ikado et al., Phys. Rev. Lett. 97 (2006) 251802 arXiv:hep-ex/0604018.

[21] W. S. Hou, Phys. Rev. D 48 (1993) 2342.

[22] G. Isidori and P. Paradisi, Phys. Lett. B 639 (2006) 499 arXiv:hep-ph/0605012.

[23] G. Isidori, F. Mescia, P. Paradisi and D. Temes, Phys. Rev. D 75 (2007) 115019 arXiv:hep-ph/0703035.

[24] A. G. Akeroyd and S. Recksiegel, J. Phys. G 29, 2311 (2003) arXiv:hep-ph/0306037.

[25] H. E. Logan and U. Nierste, Nucl. Phys. B 586 (2000) 39 arXiv:hep-ph/0004139]; C. S. Huang, W. Liao, Q. S. Yan and S. H. Zhu, Phys. Rev. D 63 (2001) 114021 [Erratum-ibid. D 64 (2001) 059902] arXiv:hep-ph/0006250.

[26] H. P. Nilles, Phys. Rept. 110 (1984) 1. 
H. E. Haber and G. L. Kane, Phys. Rept. 117 (1985) 75.

[27] D. J. H. Chung, L. L. Everett, G. L. Kane, S. F. King, J. D. Lykken and L. T. Wang, Phys. Rept. 407 (2005) 1 arXiv:hep-ph/0312378.

[28] For a recent review, see

A. Masiero and O. Vives, Ann. Rev. Nucl. Part. Sci. 51 (2001) 161 arXiv:hep-ph/0104027;

A. Masiero and O. Vives, New Jour. Phys. 4 (2002) 4.

[29] M. Olechowski and S. Pokorski, Phys. Lett. B 344 (1995) 201 arXiv:hep-ph/9407404.

[30] A. Bartl, T. Gajdosik, E. Lunghi, A. Masiero, W. Porod, H. Stremnitzer and O. Vives, Phys. Rev. D 64 (2001) 076009 arXiv:hep-ph/0103324.

[31] J. R. Ellis, K. A. Olive and Y. Santoso, Phys. Lett. B 539 (2002) 107 arXiv:hep-ph/0204192.

[32] J. R. Ellis, T. Falk, K. A. Olive and Y. Santoso, Nucl. Phys. B 652 (2003) 259 arXiv:hep-ph/0210205.

[33] V. Berezinsky, A. Bottino, J. R. Ellis, N. Fornengo, G. Mignola and S. Scopel, Astropart. Phys. 5 (1996) 1 arXiv:hep-ph/9508249].

[34] P. Nath and R. Arnowitt, Phys. Rev. D 56 (1997) 2820 arXiv:hep-ph/9701301.

[35] D. G. Cerdeno and C. Munoz, JHEP 0410 (2004) 015 arXiv:hep-ph/0405057.

[36] H. Baer, A. Mustafayev, S. Profumo, A. Belyaev and X. Tata, JHEP 0507 (2005) 065 arXiv:hep-ph/0504001.

[37] M. S. Carena, A. Menon and C. E. M. Wagner, Phys. Rev. D 76, 035004 (2007) arXiv:0704.1143 [hep-ph]].

[38] M. S. Carena, A. Menon, R. Noriega-Papaqui, A. Szynkman and C. E. M. Wagner, Phys. Rev. D 74, 015009 (2006) arXiv:hep-ph/0603106.

[39] S. Martin and M. Vaughn, Phys. Rev. D50, 2282 (1994); Y. Yamada, Phys. Rev. D 50, 3537 (1994); I. Jack, D.R.T. Jones, Phys. Lett. B333 (1994) 372.

[40] D. M. Pierce et al., Nucl. Phys. B 491 (1997) 3.

[41] W. Porod, Comput. Phys. Commun. 153 (2003) 275 arXiv:hep-ph/0301101;

[42] G. Degrassi, P. Slavich and F. Zwirner, Nucl. Phys. B 611 (2001) 403; A. Brignole, G. Degrassi, P. Slavich and F. Zwirner, Nucl. Phys. B 631 (2002) 195; Nucl. Phys. B 643 (2002) 79; A. Dedes, G. Degrassi and P. Slavich, Nucl. Phys. B 672 (2003) 144. A. Dedes and P. Slavich, Nucl. Phys. B 657 (2003) 333; B. C. Allanach, A. Djouadi, J. L. Kneur, W. Porod and P. Slavich, JHEP 0409 (2004) 044. 
[43] W. M. Yao et al. [Particle Data Group], J. Phys. G 33 (2006) 1 [hppt://pdg.lbl.gov].

[44] C. Hamzaoui, M. Pospelov and M. Toharia, Phys. Rev. D 59 (1999) 095005 arXiv:hep-ph/9807350.

[45] S. R. Choudhury and N. Gaur, Phys. Lett. B 451 (1999) 86 arXiv:hep-ph/9810307.

[46] K. S. Babu and C. F. Kolda, Phys. Rev. Lett. 84 (2000) 228 arXiv:hep-ph/9909476.

[47] G. Isidori and A. Retico, JHEP 0111 (2001) 001 arXiv:hep-ph/0110121.

[48] C. Bobeth, T. Ewerth, F. Kruger and J. Urban, Phys. Rev. D 66 (2002) 074021 arXiv:hep-ph/0204225.

[49] P. H. Chankowski and L. Slawianowska, Phys. Rev. D 63 (2001) 054012 [arXiv:hep-ph/0008046].

[50] A. J. Buras, P. H. Chankowski, J. Rosiek and L. Slawianowska, Phys. Lett. B 546 (2002) 96 arXiv:hep-ph/0207241.

[51] A. J. Buras, P. H. Chankowski, J. Rosiek and L. Slawianowska, Nucl. Phys. B 659 (2003) 3 arXiv:hep-ph/0210145.

[52] G. L. Kane, C. Kolda and J. E. Lennon, arXiv:hep-ph/0310042.

[53] A. Dedes and B. T. Huffman, Phys. Lett. B 600 (2004) 261 arXiv:hep-ph/0407285.

[54] E. Lunghi, W. Porod and O. Vives, Phys. Rev. D 74 (2006) 075003 arXiv:hep-ph/0605177.

[55] J. R. Ellis, S. Heinemeyer, K. A. Olive, A. M. Weber and G. Weiglein, JHEP 0708 (2007) 083 arXiv:0706.0652 [hep-ph]].

[56] A. Dedes, H. K. Dreiner and U. Nierste, Phys. Rev. Lett. 87 (2001) 251804 arXiv:hep-ph/0108037.

[57] S. Baek, P. Ko and W. Y. Song, JHEP 0303 (2003) 054 arXiv:hep-ph/0208112.

[58] A. Freitas, E. Gasser and U. Haisch, Phys. Rev. D 76 (2007) 014016 arXiv:hep-ph/0702267.

[59] A. Bartl, W. Majerotto, W. Porod and D. Wyler, Phys. Rev. D 68 (2003) 053005 arXiv:hep-ph/0306050 and references therein.

[60] F. Ambrosino et al. [KLOE Collaboration], Phys. Lett. B 632 (2006) 76 arXiv:hep-ex/0509045.

[61] Flavianet kaon WG, http://www.lnf.infn.it/wg/vus/,

arXiv:0801.1817 [hep-ph].

[62] R. Dermisek, S. Raby, L. Roszkowski and R. Ruiz De Austri, JHEP 0304 (2003) 037 arXiv:hep-ph/0304101. 
[63] R. Dermisek, S. Raby, L. Roszkowski and R. Ruiz de Austri, JHEP 0509 (2005) 029 arXiv:hep-ph/0507233.

[64] S. Baek, Y. G. Kim and P. Ko, JHEP 0502 (2005) 067 arXiv:hep-ph/0406033.

[65] G. F. Giudice and R. Rattazzi, Phys. Rept. 322 (1999) 419 [arXiv:hep-ph/9801271.

[66] L. Randall and R. Sundrum, Nucl. Phys. B 557, 79 (1999) arXiv:hep-th/9810155.

[67] G. F. Giudice, M. A. Luty, H. Murayama and R. Rattazzi, JHEP 9812 (1998) 027 arXiv:hep-ph/9810442.

[68] R. Kitano, G. D. Kribs and H. Murayama, Phys. Rev. D 70 (2004) 035001 arXiv:hep-ph/0402215.

[69] R. Hodgson, I. Jack, D. R. T. Jones and G. G. Ross, Nucl. Phys. B 728 (2005) 192 arXiv:hep-ph/0507193.

[70] S. Schael et al. [ALEPH Collaboration], Eur. Phys. J. C 47 (2006) 547 arXiv:hep-ex/0602042].

[71] A. Bartl, W. Majerotto and W. Porod, Z. Phys. C 64 (1994) 499 [Erratum-ibid. C 68 (1995) $518]$.

[72] F. Borzumati and A. Masiero, Phys. Rev. Lett. 57, 961 (1986);

[73] J. Hisano, T. Moroi, K. Tobe, M. Yamaguchi and T. Yanagida, Phys. Lett. B 357 (1995) 579 [hep-ph/9501407].

[74] A. Masiero, S. K. Vempati and O. Vives, Nucl. Phys. B 649, 189 (2003) hep-ph/0209303]; New J. Phys. 6, 202 (2004) hep-ph/0407325; L. Calibbi, A. Faccia, A. Masiero and S. K. Vempati, Phys. Rev. D 74 (2006) 116002 hep-ph/0605139].

[75] J. Hisano and K. Tobe, Phys. Lett. B 510 (2001) 197 hep-ph/0102315.

[76] K. S. Babu and C. Kolda, Phys. Rev. Lett. 89 (2002) 241802 hep-ph/0206310.

[77] M. Sher, Phys. Rev. D 66 (2002) 057301 arXiv:hep-ph/0207136; A. Brignole and A. Rossi, Nucl. Phys. B 701 (2004) 3 arXiv:hep-ph/0404211.

[78] A. Dedes, J. R. Ellis and M. Raidal, Phys. Lett. B 549 (2002) 159 arXiv:hep-ph/0209207.

[79] R. Kitano, M. Koike, S. Komine and Y. Okada, Phys. Lett. B 575 (2003) 300 arXiv:hep-ph/0308021.

[80] A. Brignole and A. Rossi, Phys. Lett. B 566 (2003) 217 arXiv:hep-ph/0304081.

[81] P. Paradisi, JHEP 0602, 050 (2006) hep-ph/0508054); P. Paradisi, JHEP 0608, 047 (2006) hep-ph/0601100. 